\title{
The presence of two distinct prolactin receptors in seabream with different tissue distribution patterns, signal transduction pathways and regulation of gene expression by steroid hormones
}

\author{
Xigui Huang ${ }^{1,2, *}$, Baowei Jiao ${ }^{2,3, *}$, Chun Kit Fung ${ }^{2, *}$, Yong Zhang ${ }^{4, *}$, Walter K K Ho ${ }^{2}$, Chi Bun Chan ${ }^{2}$, \\ Haoran Lin ${ }^{4}$, Deshou Wang ${ }^{1}$ and Christopher H K Cheng ${ }^{2}$ \\ ${ }^{1}$ Key Laboratory of Eco-environments in Three Gorges Reservoir Region (Ministry of Education), Key Laboratory of Aquatic Science of Chongqing, School of Life \\ Science, Southwest University, Chongqing 400715, China \\ ${ }^{2}$ Department of Biochemistry and ${ }^{3}$ The Environmental Science Programme, The Chinese University of Hong Kong, Shatin, N.T., Hong Kong, China \\ ${ }^{4}$ State Key Laboratory of Biocontrol, Institute of Aquatic Economic Animals, and the Guangdong Province Key Laboratory for Aquatic Economic Animals, Sun \\ Yat-Sen University, Guangzhou 510275, China \\ (Requests for offprints should be addressed to D Wang; Email: wdeshou@swu.edu.cn; C H K Cheng; Email: chkcheng@cuhk.edu.hk) \\ *(X Huang, B Jiao, C K Fung and Y Zhang contributed equally to this work)
}

\begin{abstract}
Two prolactin receptors (PRLRs) encoded by two different genes were identified in the fugu and zebrafish genomes but not in the genomes of other vertebrates. Subsequently, two cDNA sequences corresponding to two PRLRs were identified in black seabream and Nile tilapia. Phylogenetic analysis of PRLR sequences in various vertebrates indicated that the coexistence of two PRLRs in a single species is a unique phenomenon in teleosts. Both PRLRs in teleosts (the classical one named as PRLR1, the newly identified one as PRLR2) resemble the long-form mammalian PRLRs. However, despite their overall structural similarities, the two PRLR subtypes in fish share very low amino acid similarities (about 30\%), mainly due to differences in the intracellular domain. In particular, the Box 2 region and some intracellular tyrosine residues are missing in PRLR2. Tissue distribution study by real-time PCR in black seabream (sb) revealed that both receptors (sbPRLR 1 and sbPRLR 2 ) are widely expressed in different tissues. In gill, the expression level of sbPRLR2 is much higher than that of sbPRLR1. In the intestine, the expression of sbPRLR1 is higher than that of sbPRLR2. The expression levels of both receptors are relatively low in most other tissues, with sbPRLR1 generally higher than sbPRLR2. The sbPRLR 1 and sbPRLR 2 were functionally expressed in
\end{abstract}

cultured human embryonic kidney 293 cells. Both receptors can activate the $\beta$-casein and c-fos promoters; however, only sbPRLR1 but not sbPRLR2 can activate the Spi promoter upon receptor stimulation in a ligand-specific manner. These results indicate that both receptors share some common functions but are distinctly different from each other in mobilizing post-receptor events. When challenged with different steroid hormones, the two PRLRs exhibited very different gene expression patterns in the seabream kidney. The sbPRLR1 expression was up-regulated by estradiol and cortisol, whereas testosterone had no significant effect. For sbPRLR2, its expression was down-regulated by estradiol and testosterone, while cortisol exerted no significant effect. The $5^{\prime}$-flanking regions of the $s P R L R 1$ and $s b P R L R 2$ genes were cloned and the promoter activities were studied in transfected GAKS cells in the absence or presence of different steroid hormones. The results of the promoter studies were in general agreement with the in vivo hormonal regulation of gene expression results. The $s b P R L R 1$ gene promoter activity was activated by estradiol and cortisol, but not by testosterone. In contrast, the $s b P R L R 2$ gene promoter activity was inhibited by estradiol, cortisol, and testosterone.

Journal of Endocrinology (2007) 194, 373-392

\section{Introduction}

Prolactin (PRL) is recognized as the most versatile pituitary hormone among diverse species (Manzon 2002). In fish, various physiological functions have been attributed to PRL, such as enhancement of immune functions (Harris \& Bird 2000), pigment dispersion in the tegumentary chromatophores (Kitta et al. 1993), synergism with production of steroid hormones in the gonads (de Ruiter et al. 1986), and reproduction (Cavaco et al. 2003).

PRL exerts its actions via specific PRL receptor (PRLR) which belongs to the cytokine class I receptor superfamily (Bole-Feysot et al. 1998). Besides mammals and other tetrapods, PRLRs have also been cloned and characterized in different fish species (Sandra et al. 1995, Tse et al. 2000, Le Rouzic et al. 2001, Santos et al. 2001, Lee et al. 2006). 
In mammals, there are long, intermediate, and short PRLR forms that are generated by alternative splicing and promoter usage (Freeman et al. 2000). These isoforms have identical extracellular domains but differ in the length and composition of their intracellular domains as well as in their signal transduction mechanisms (Bole-Feysot et al. 1998). However, the presence of well-characterized multiple forms of PRLR in teleost has not been reported. The fact that there are two different PRLs in tilapia (PRL177 and PRL188) and their different physiological functions imply the possible existence of more than one PRLR in tilapia (Sandra \& Prunet 1998). Yet no other forms of PRLR in Nile tilapia have been identified (Sandra et al. 1995). In other teleosts, PRLR transcripts of different sizes have been found by northern blot analysis in seabream (Santos et al. 2001) and goldfish (Tse et al. 2000), but their identities have not been ascertained.

In order to identify the presence of multiple genes for PRLR in a single species, in silico data mining was carried out in different fish and tetrapod genomes. Such search results indicated the presence of two putative $P R L R$ genes in teleosts but not in other vertebrates. To further substantiate the coexistence of two PRLRs in a single fish species, we have cloned two types of PRLR cDNAs in black seabream (sbPRLR1 and sbPRLR2). We have also cloned a novel PRLR subtype (ntPRLR2) from Nile tilapia in which the other PRLR subtype (ntPRLR1) is already known. Subsequently, a phylogenetic tree was constructed from all the available PRLR sequences. The results clearly indicated that the PRLR sequences could be grouped into two different clades, namely PRLR1 and PRLR2. Using black seabream (Acanthopagrus schlegeli) as the model organism, we have investigated the tissue distribution and signal transduction mechanisms of the two PRLRs, as well as their in vivo regulation of gene expression by steroid hormones. We have also cloned the promoters of these two genes in black seabream and studied their responsiveness to steroid hormone challenge in transfected cultured cells.

\section{Materials and Methods}

\section{Experimental animals}

Black seabream (body length $22.5 \pm 0.3 \mathrm{~cm}$, body weight $235 \pm 11 \mathrm{~g}$ ) and Nile tilapia (body length $20 \pm 4 \mathrm{~cm}$, body weight $200 \pm 20 \mathrm{~g}$ ) of both sexes were purchased from a local fish farm. All experiments were conducted in accordance with guidelines as established by the University Committee on the use and care of laboratory animals at the Chinese University of Hong Kong.

\section{Data mining and phylogenetic analysis}

Genomic contigs corresponding to the putative $P R L R$ genes were identified by searching the genome databases (Ensembl genome browser http://www.ensembl.org/index.html and NCBI database http://www.ncbi.nlm.nih.gov/ using TBlastN algorithm) of zebrafish (Danio rerio, fish), fugu (Tetraodon nigroviridis, fish), chicken (Gallus gallus, avian), Xenopus (Xenopus tropicalis, amphibian), and mouse (Mus musculus, mammal; Jiao et al. 2006). Nucleotide and amino acid (aa) sequence editing, comparison, and alignment were performed using Editseq and Megalign from the DNASTAR package (DNASTAR, Madison, WI, USA). The multiple alignment software ClustalX (Thompson et al. 1997) was employed to construct the phylogenetic tree by using the neighbor-joining method. Treeview was used to display the phylogenetic tree.

\section{Molecular cloning of sbPRLR1 and sbPRLR2 from black seabream and ntPRLR2 from Nile tilapia}

According to the known fish PRLR1 cDNA sequences and the predicted PRLR2 cDNA sequences in fugu and zebrafish, degenerate primers were designed for cloning the PRLRs in black seabream and Nile tilapia. A plasmid cDNA library of average insert size larger than $2 \mathrm{~kb}$ that was constructed from poly(A) ${ }^{+}$mRNA prepared from seabream gill using the pSPORT1 vector (Invitrogen) was used as the PCR template to obtain a fragment each of sbPRLR1 and sbPRLR2. Methods of rapid amplification of cDNA ends (RACE) were applied to obtain the full-length sequences of sbPRLR1 and sbPRLR2. Seabream gill total RNA was extracted according to the manufacturer's protocol (Roche Applied Science). $5^{\prime}$ and $3^{\prime}$ RACE were performed using the $5^{\prime}$ and $3^{\prime}$ RACE amplification kits (Invitrogen) respectively. The full-length cDNA clones were confirmed by DNA sequencing in at least three different individual clones. For the Nile tilapia PRLR2, the whole cloning procedure was similar to that of sbPRLRs, except that the tilapia gill tissues were used to prepare the first strand cDNA as the template for PCR.

For all the PCRs in the present study, reactions were carried out with an initial denaturation at $94^{\circ} \mathrm{C}$ for $3 \mathrm{~min}$, followed by 35 cycles of $94^{\circ} \mathrm{C}$ for $30 \mathrm{~s}, 52-58{ }^{\circ} \mathrm{C}$ for $30 \mathrm{~s}$, and $72{ }^{\circ} \mathrm{C}$ for $1-1.5 \mathrm{~min}$. The reaction was ended by a further $10 \mathrm{~min}$ at $72{ }^{\circ} \mathrm{C}$. The amplicons were resolved on a $1 \%$ agarose gel and the target DNA fragment was purified by the QIAquick Gel Extraction Kit (Qiagen). The fragment was then cloned into pCR2.1-TOPO vector (Invitrogen) and sequenced by an ABI 3700 sequencer (Applied Biosystems, Foster City, CA, USA). Sequence information of all the primers used in the present study is listed in Table 1.

Functional expression of sbPRLR1 and sbPRLR2 in cultured eukaryotic cells

Functional expression is a prerequisite for demonstrating the biological activities of cloned sequences (Chan \& Cheng 
Table 1 The primer sequences used in the present study

Sequences $\left(5^{\prime}\right.$ to $\left.3^{\prime}\right)$

\section{Primer}

sbPRLR1-F1

sbPRLR1-F2

SbPRLR1-R6

sbPRLR1-R2

SbPRLR1-F8

sbPRLR-UTR-R1

SbPRLR-UTR-R2

sbPRLR1-Race-R1

sbPRLR1-Race-R2

SBPRLR1-UTR-F

SbPRLR1-UTR-R

sbPRLR2-F4

sbPRLR2-R4

sbPRLR2-F6

sbPRLR2-Race-R1

sbPRLR2-Race-R2

SbPRLR2-UTR-F1

SbPRLR2-UTR-R

ntPRLR2-F1

ntPRLR2-R1

ntPRLR2-F2

ntPRLR2-F3

ntPRLR2-R2

ntPRLR2-R3

sbPRLR1-sense

sbPRLR1-antisense

sbPRLR2-sense

sbPRLR2-antisense

SbPRLR1-ORF-R

SbPRLR2-ORF-R

c-myc-F

C-myc-R

SbPRLR1-PR1

SbPRLR1-PR2

SbPRLR1-PR3

SbPRLR2-PR1

SbPRLR2-PR2

SbPRLR2-PR3

PR1F1

PR1F2

PR1R1

PR2F1

PR2R1

AP

AAP

AUAP

T7

SP6

AP-1

AP-2

sb-actin-F

sb-actin-R
TCT CCW GAG AAR GAG ACC TTC

TGG GTC AAC TAC AAC ATC ACT GT

ACY YCT TTG ACC TTG CTG TAG T

TCS TCW GAC TTK CCR CTC TTG AG

TCA ATG AGG AGG GTA TGG TGC

ACA TAK CCA CTY ACT GCC AG

TCC ASA MCT GCC WGT TYG G

TGC ATG CGT GAA GAC TAG CAG CA

GAT AAC TTC AAG GAC TTT CC CAT

GGA GAG ACT GAA AGC TGC TGT CA

CCC ACC TGC CAG TTT GGG CAC CTA

ATT GGA GGG AGT GCA CGA GTGT

GTG CAG GGA TGG TAT CTA TAT AT

CTC CTG TTC CTG GTC CAA AGAT

TTC CCT GGT GGA GAC GAG CTG

CTG CAC ACA GCA GCA CAA GCA C

CAG CTG GAC ACA CTG CGG GAC A

ACT ACA CAA TAC CAT CAG CCT

GKT CCC CHG AVA ARG AGA C

GGA CCA GGR ACW GGD GGC AG

CAG CCT CTC TCT GAT TCC GTT GC

TAT TCT GCC CCC TGT TCC TGG TC

CAA GTC AAA AGT AAG GGG GTC TG

GGA GTG TGC TGT GTG GTA GTC TG

CGG TCT TCT GTG CCT TCA TCT

CAC CAG CAA GTC CTC GTA GTT AG

TCT GCC TCC TGT TCC TGG TC

GAA GCC AGC AGG AAT AAT CAA G

TAT AGC GGC CGC GTA GGT AGG CAG TGT GAA A

TAT AGC GGC CGC CAT TAA AGG TGA TGC AGG GA

TAT AGC GGC CGC TGA GCA GAA GCT GAT CTCA

TAT ATC TAG ACT ACA GAT CCT CCT CTG AGA T

CGG CTC CCA GAA GAA CGA ACT GAA CT

CTC CCA GAA GAA CGA ACT GAA CTC ACC

GTC AGT AGT TAC TTT GCA TCA GAG C

ACT CCA CTG AGT CAC TCA ACT CCA TGT

TCA CTC AAC TCC ATG TTG CTG CTG TC

GCA CAG CAG CAA AGA TTT GTA CAC AG

TCG TGA GAT GTT TAG GAG AAT GC

TAT AAC GCG TGG AGA ATG CTG CAG CTC TGT (Mlu I)

TAT AAG ATC TTC CAC CGC CTC TCA GTC CGC (Bgl II)

GGG TGA GCT CGA ATC TTA AGG GAT CTC TCT

TGG GAA GCT TCA CCT GAG GTG GAG TAT GTA

GGC CAC GCG TCG ACT AGT AC(T)16

GGC CAC GCG TCG ACT AGT AC GGG GGG GGG G

GGC CAC GCG TCG ACT AGT AC

TAA TAC GAC TCA CTA TAG GG

ATT TAG GTG ACA CTA TAG AA

GTA ATA CGA CTC ACT ATA GGG C

ACT ATA GGG CAC GCG TGG T

ACC CAG ATC ATG TTC GAG ACC

ATG AGG TAG TCT GTG AGG TCG
Purpose

cDNA fragment PCR

RACE

Full-length PCR amplification

cDNA fragment PCR

RACE

Full-length PCR amplification

cDNA fragment PCR

RACE

Real-time PCR

c-myc tag PCR

PRLR1 genome walking

PRLR2 genome walking

sbPRLR1 promoter cloning

sbPRLR2 promoter cloning

RACE

Library PCR

Genome walking

Actin PCR amplification
2004, Chan et al. 2004). The full-length sbPRLR1 and sbPRLR2 cDNA clones were functionally expressed in cultured human embryonic kidney (HEK293) cells (American Type Culture Collection, Manassas, VA, USA) and their biological activities studied. HEK293 cells were transiently transfected with $50 \mathrm{ng}$ pcDNA3.1 vector (Invitrogen) carrying the entire coding region of either sbPRLR1 or
sbPRLR2 between the EcoR V and Not I sites. In order to compare the transfection efficiency and protein expression level of the two receptor constructs, a c-myc tag was attached to the $\mathrm{C}$ terminus of both receptors by taking advantage of the Not I and Xba I sites of the pcDNA3.1 vector. This would allow western analysis of the expression levels of the two receptor proteins in the transfected cells using specific 
antibodies against the c-myc tag. The functionality of the expressed receptors in the transfected cells was evaluated by the ability of the receptors to transactivate certain gene promoters in a reporter gene assay format. Briefly, $500 \mathrm{ng}$ luciferase reporter plasmid containing either the rat $\beta$-casein promoter in pLucDSS or the human $\mathrm{c}$-fos promoter in pFL711 or the rat serine protease inhibitor (Spi 2.1) promoter in pGL2, were co-transfected together with either one of the two PRLR expression constructs. After $6 \mathrm{~h}$ of transfection, purified salmon growth hormone (sGH), PRL (sPRL), or somatolactin (sSL) was added. The cells were then incubated with the respective hormones for a further $20 \mathrm{~h}$. Afterwards, luminescence was measured using a Lumat LB 9501 luminometer (EG\&G, Berthold, Germany) and activities of both the firefly luciferase and the Renilla luciferase were measured sequentially from a single sample using the Dual Luciferase Kit (Promega).

\section{Western blot}

Western blot analysis was carried out according to a standard protocol (Sambrook et al. 1989). Briefly, HEK293 cells were lysed at $4{ }^{\circ} \mathrm{C}$. The BCA protein assay kit (Pierce, Rockford, IL, USA) was used to determine the protein concentrations of the cell lysates. Equal amounts of protein were loaded onto polyacrylamide gels, electrophoresed, and transferred onto nitrocellulose membranes (Schleicher \& Schuell, Keene, NH, USA) using a semi-dry electroblotter (Owl Scientific, Woburn, MA, USA). After transfer, the membranes were blocked overnight in 5\% dry milk and probed for protein expression using the anti-c-myc antibody (Promega) and then with goat anti-mouse IgG peroxidase-conjugated secondary antibodies (Promega). The bands were stained and viewed on an alpha imager (Alpha Innotech Co., San Leandro, CA, USA).

\section{Expression studies of sbPRLR 1 and sbPRLR2 in seabream tissues by real-time PCR}

The sbPRLR1 and sbPRLR2 transcript levels in different seabream tissues were measured by real-time PCR using $\beta$-actin as the internal control. Gene-specific primers for sbPRLR1, sbPRLR2, and $\beta$-actin are listed in Table 1. The primers were designed on different exons so that any genomic contamination could be detected. The primers for sbPRLR1 and sbPRLR2 amplified two specific amplicons of 215 and $235 \mathrm{bp}$, respectively.

First strand cDNA was synthesized from $3 \mu \mathrm{g}$ total RNA prepared from seabream tissues using oligo- $\mathrm{dT}_{18}$ as primer and MMLV-H ${ }^{-}$reverse transcriptase as enzyme (GeneSys, Borehamwood, Herts, England). PCR amplification and analysis were performed on an MJ Real-time Detection System (MJ, Hercules, CA, USA). The final volume of the reaction was $25 \mu \mathrm{l}$, using the ABI SYBR Green Supermix (ABI, Warrington, UK). The specificity of the SYBR Green PCR signal was confirmed by melting curve analysis and agarose gel electrophoresis. For each particular gene, the efficiency of the PCR
(91-98\%) was the same for serial dilutions of the standards and samples. Agarose gel electrophoresis exhibited a single band of the expected size, and all amplified products showed a single melting peak on real-time PCR. The threshold cycle $(\mathrm{Ct})$ was defined as the fractional cycle number by the method of global minimum. The ratio change in the target gene relative to $\beta$-actin control gene was determined by the $2^{-\Delta \Delta \mathrm{Ct}}$ method (Livak \& Schmittgen 2001, Vong et al. 2003). There was some minor but insignificant variation in the $\mathrm{Ct}$ values of $\beta$-actin in the different tissues as well as in the samples of the hormonal treatment study described in the next section, indicating the validity of using $\beta$-actin as the internal control in our study. Other studies have also reported on the use of $\beta$-actin as the internal control in gene expression studies in fish (Celius et al. 2000, Yada et al. 2005).

Hormonal regulation of sbPRLR1 and sbPRLR2 gene expression in seabream kidney in vivo by real-time PCR

Black seabreams (sexually matured, gonad regressed, body length $22 \cdot 5 \pm 0 \cdot 3 \mathrm{~cm}$, body weight $235 \pm 11 \mathrm{~g}$ ) were acclimatized for at least 1 month before the experiment. Throughout the study, fish were kept in fully aerated seawater and were exposed to natural photoperiod at ambient temperature $\left(24-29^{\circ} \mathrm{C}\right)$ in May, 2004. Fish were fed with commercially available fish pellet diet until satiety during the experimental period. Fish were injected with either cortisol (5 $\mu \mathrm{g} / \mathrm{g}$; Sigma), $17 \beta$-estradiol ( $\mathrm{E}_{2} ; 2 \mu \mathrm{g} / \mathrm{g}$; Sigma), or testosterone $(2 \mu \mathrm{g} / \mathrm{g}$; Sigma), intraperitoneally once daily for 4 days. Cortisol, $\mathrm{E}_{2}$, and testosterone were dissolved in olive oil. The injections were performed at $1000 \mathrm{~h}$ every day and the tissues were collected from the fish at $1500 \mathrm{~h}$. on the day of killing. Olive oil was used as the vehicle in the control groups. The fish were then killed by decapitation and tissues were kept at $-70{ }^{\circ} \mathrm{C}$ after freezing in liquid nitrogen. The transcript levels of sbPRLR1 and sbPRLR 2 were measured by real-time PCR as described in the section on tissue distribution studies.

\section{Isolation of SbPRLR1 and SbPRLR2 $5^{\prime}$-flanking regions and preparation of promoter reporter constructs}

The sbPRLR1 and sbPRLR2 transcription start sites were determined by $5^{\prime}$ RACE. Gene-specific primers were designed at the most $5^{\prime}$ ends of both cDNAs. Isolation of the $5^{\prime}$-flanking regions of the $s b P R L R$ genes was achieved using the Universal Genome Walker Kit (BD Biosciences Clontech). Five Genome Walker libraries (Dra I, EcoR V, $P v u$ II, Stu I and Sca I) were constructed according to the manufacturer's instructions (Yeung et al. 2004, 2006).

The $s P R R L R 1$ gene fragment was obtained from two primers containing two restriction enzyme cutting sites, namely $M l u \mathrm{I}$ and $B g l \mathrm{II}$, and then the product was cloned into the pCR2.1TOPO vector (Invitrogen). The TOPO vector with the cloned gene fragment was digested with $M l u \mathrm{I}$ and $B g l \mathrm{II}$ and cloned into the $M l u \mathrm{I} / B g l$ II sites of the pGL3-basic vector. The $s b P R L R 2$ gene fragment was directly inserted into the Sac I/Hind III sites of the pGL3-basic vector. All the plasmid DNA used in the present study for transfection experiments was prepared using 
the QIAfilter Plasmid Maxi Kit (Qiagen). The primer sequences and enzyme cutting sites are listed in Table 1.

\section{Gene promoter assays}

The culture media used for cell culture experiments were obtained from Invitrogen. GAKS cells are goldfish scale fibroblast cells from the RIKEN Bioresource Center (Ibaraki, Japan). This fish cell line expresses glucocorticoid receptor (GR) and androgen receptor (AR), but not estrogen receptor (ER) as indicated by RT-PCR using gene-specific primers (data not shown). Transient transfection of the GAKS cells was carried out using the Lipofectamine Reagent (Invitrogen). The cells were seeded onto 24-well culture plates (Iwaki) at a density of $1.5 \times$ $10^{5}$ cells/well $24 \mathrm{~h}$ prior to transfection. The GAKS cells were transfected with $0.5 \mu \mathrm{g}$ of either the $s b P R L R 1$ or $s b P R L R 2$ gene promoter construct in pGL3-basic, and 0.05 $\mu \mathrm{g}$ pRLCMV vector containing the Renilla luciferase reporter gene in $250 \mu \mathrm{l}$ serum-free medium. After $6 \mathrm{~h}$ of transfection, the medium was aspirated away and replaced with complete growth medium. The transfected cells were treated with different steroid hormones, namely $\mathrm{E}_{2}$, cortisol, and testosterone in serum-free medium for $24 \mathrm{~h}$. For the cells treated with $\mathrm{E}_{2}, 50 \mathrm{ng}$ goldfish ER $\alpha$ was also co-transfected and the medium was phenol-red free. Then the cells were washed and lysed for determination of luciferase activities. The promoter activity was normalized against the Renilla luciferase activity and expressed as the percentage increase relative to the activity of the promoterless pGL3-basic vector.

\section{Data analysis}

All data were expressed as mean values \pm s.E.M. Data were considered statistically significant at $P<0 \cdot 05$ using either one-way analysis of variance (ANOVA) or unpaired $t$-test.

\section{Results}

Data mining and molecular cloning of PRLR 1 and PRLR2 in different fish species

Recently, several vertebrate genome databases such as zebrafish, fugu, African clawed frog, chicken, mouse, and human have become available. Using these genome information, we conducted bioinformatics analysis to look for PRLR genes in different organisms. Briefly, the available PRLR protein sequences were used to blast the different genomes using the TblastN method. Then the blast hits with the highest scores and lowest expect value were singled out for identifying the coding regions of the respective PRLR. Such search results indicated the presence of two PRLR genes in the fish genome, a phenomenon unique for teleosts.

By RT-PCR and RACE techniques, two PRLR cDNAs were obtained from black seabream. The full-length sbPRLR1 cDNA contains $2069 \mathrm{bp}$ encompassing a $5^{\prime}$-untranslated region (UTR) of $182 \mathrm{bp}$, a coding region of $1866 \mathrm{bp}$ and a partial $3^{\prime}-\mathrm{UTR}$. The coding region encodes a protein of 621 aa with a putative single transmembrane domain (Fig. 1). The sbPRLR2 contains 2751 bp encompassing a $5^{\prime}-\mathrm{UTR}$ of $158 \mathrm{bp}$, a coding region of $1614 \mathrm{bp}$, and a $3^{\prime}$-UTR of $979 \mathrm{bp}$ together with a poly (A) tail. The coding region encodes a protein of 537 aa (Fig. 2). Several characteristic landmarks of PRLR including the WS motif, two pairs of cysteine residues in the extracellular region, and a conserved intracellular Box 1 motif were found in both sbPRLR1 and sbPRLR2. However, unlike sbPRLR1, the absence of a typical Box 2 in sbPRLR2 was noted. In addition, we have also obtained a PRLR2 cDNA in Nile tilapia containing $2011 \mathrm{bp}$, encompassing a 5'-UTR of $217 \mathrm{bp}$, a coding region of $1590 \mathrm{bp}$ encoding a protein of 529 aa, and a 3'-UTR of 204 bp (Fig. 3). Again, the Box 2 region was missing in this sequence. Despite the overall similarities between PRLR1 and PRLR2, distinct differences were found in their intracellular regions where several deletions were identified in PRLR2 (Fig. 4). The overall sequence similarity between PRLR1 and PRLR2 is rather low, being $28-40 \%$ at the aa level (Table 2). The sequence similarity among the PRLR2s themselves is also low, being $30-46 \%$ at the aa level (Table 3).

\section{PRLR1 and PRLR2 alignment and phylogenetic analysis}

A multiple alignment of PRLR 1 and PRLR 2 sequences was performed in the four fish species in which both PRLR 1 and PRLR2 sequences are known to date, viz. fugu, zebrafish, Nile tilapia, and black seabream. As shown in Fig. 4, PRLR1 and PRLR2 are very similar in the extracellular region, including the four conserved cysteine residues and the WS motif. On the other hand, remarkable differences were found in the intracellular region. Notably, PRLR 2 does not possess the typical Box 2 motif and several other missing regions in the intracellular domain are also found. Using the available PRLR sequences to construct a phylogenetic tree, the results clearly showed that there are two clades of PRLR representing two different subtypes of PRLR in fish (Fig. 5). The PRLR1 clade encompasses the sequences previously reported in a number of fish species including the goldfish (Tse et al. 2000), Nile tilapia (Sandra et al. 1995), halibut (Higashimoto et al. 2001), rainbow trout (Prunet et al. 2000), carp (San Martin et al. 2006) as well as the black seabream PRLR1 and fugu PRLR1. The PRLR2 clade, unique to teleosts, encompasses the previously reported gilthead seabream PRLR (Santos et al. 2001), fugu PRLR2 (Lee et al. 2006), as well as the black seabream PRLR2, Nile tilapia PRLR2, and zebrafish PRLR2.

\section{Functional expression of sbPRLR 1 and sbPRLR2 in transfected} HEK293 cells

As shown in Fig. 6, when sbPRLR1 and sbPRLR2 were individually expressed in cultured HEK293 cells, receptor 
CGACTTCTTGTGGCAGACGCTCATGAGTGTACCATCGTCCCACCAGCCCGGTAAACCGGTTACACAAGTCGGTGAGTTCAGTTCGTTCT TCTGGGAGCCGCCAGTGGACCCAGCACGGCCAGAACACCGAGGGAGAGACTGAAAGCTGCTGTCAGCTGAAGCTGAGGCTTTGTGGTGCCACG ATG AGg AAA GTC CTT GAA GTT ATC CTG CTG TTG TTG CTG CTA GTC TTC ACG CAT GCA AAG GGA ACG CGC TAC AGC 75 $\begin{array}{lllllllllllllllllllllllllll}M & R & K & V & L & E & V & I & \text { L } & \text { L } & \text { L } & \text { L } & \text { L } & \text { L } & \text { V } & \text { F } & \text { T } & \text { H } & \text { A } & \text { K } & G & \text { T } & R & Y & S & 25\end{array}$ CCT CCG GGG AAG CCT GCG CTG ACC AGA TGT CGT TCC CCT GAA AAA GAG ACC TTC ACC TGC TGG TGG GAG CCG GGA 150

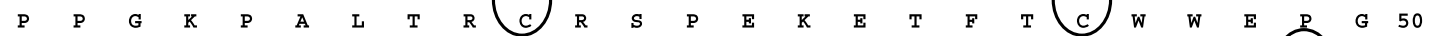
TCT GAC GGG GGG CTG CCC ACC ACA TAC GC CTG TAC TAT CGC AAA GAG AGC TCT GAC ACG GTG AAC GAA TGT CCT 225 $\begin{array}{lllllllllllllllllllllllllllllllll}S & D & G & G & \text { L } & P & T & T & Y & A & L & Y & Y & R & K & E & S & S & D & T & V & N & E & C & P & 75\end{array}$ GAC TAC CGC ACG GCC GGA GAG AAC TCC TGC TTC TTT AAC AAG AAC GAC ACA TCC ATC TGG GTC AAC TAC AAC ATC 300

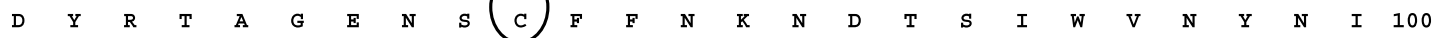
ACC GTG GTG GCC ACC AAC GCG CTG GGA AGA ACC TTC TCC GAC CCC GTG GAT ATA GAC GTG GTG TAC ATC GTC AAG 375 $\begin{array}{llllllllllllllllllllllllllllll}\text { T } & \text { V } & \text { V } & \text { A } & \text { T } & \text { N } & \text { A } & \text { L } & \text { G } & \text { R } & \text { T } & \text { F } & \text { S } & \text { D } & \text { P } & \text { V } & \text { D } & \text { I } & \text { D } & \text { V } & \text { V } & \text { Y } & \text { I } & \text { V } & K & 125\end{array}$ CCC AAT CCT CCA GAG CAG GTA GCC GTC ACT GTG ATG GAG GAC AAG GGC TGG CCC TTC ATC CGG GTG TCA TGG GAG 450 $\begin{array}{lllllllllllllllllllllllllllll}P & N & P & P & E & Q & V & A & V & T & V & M & E & D & K & G & \text { W } & P & F & I & R & V & S & W & E & 150\end{array}$ CCG CCG CAT AAG GCC GAC ACC CGC TCC GGT TGG ATC ACG CTC ATG TAC GAG ATC CGC GTC AAG TTG GAG GAG GAA 525 $\begin{array}{lllllllllllllllllllllllllll}P & P & H & K & A & D & T & R & S & G & \text { W } & \text { I } & \text { T } & \text { L } & \text { M } & \text { Y } & \text { E } & \text { I } & \text { R } & \text { V } & \text { K } & \text { L } & E & E & E & 175\end{array}$ AAC GAC TGG GAG ACG CAC CTC GCC GGC CAG CAG AAG ATG TTT AAC ATC TTC AGC CTG CGG TCA GGC GGC ACG TAT 600 $\begin{array}{llllllllllllllllllllllllllll}\mathbf{N} & \mathrm{D} & \mathrm{W} & \mathrm{E} & \mathrm{T} & \mathrm{H} & \mathrm{L} & \mathrm{A} & \mathrm{G} & \boldsymbol{Q} & \mathcal{Q} & \mathrm{K} & \mathrm{M} & \mathrm{F} & \mathrm{N} & \mathrm{I} & \mathrm{F} & \mathrm{S} & \mathrm{L} & \mathrm{R} & \mathrm{S} & \mathrm{G} & \mathrm{G} & \mathrm{T} & \mathrm{Y} & 200\end{array}$ CTT GTT CAG GTG CGC TGT AAG CCC GAT CAC GGC TTC TGG AGC GAG TGG AGT TCC TCT TCC TAC GTC AAA GTC CCC 675 $\begin{array}{lllllllllllllllllllllllllllll}\text { L } & \text { V } & \& & \text { V } & R & \text { C } & \text { K } & \text { P } & \text { D } & \text { H } & \text { G } & \text { F } & \text { W } & \text { S } & \text { E } & \text { W } & \text { S } & \text { S } & \text { S } & \text { S } & \text { Y } & \text { V } & \text { K } & \text { V } & P & 225\end{array}$ GAC TAT TTC CAT CGg GAG AAg TCC GTG TGG ATC CTC ATC ACG GTC TTC TGT GCC TTC ATC TTC CTC ATC GTC ACC 750 $\begin{array}{llllllllllllllllllllllllllll}D & \text { Y } & \text { F } & \text { H } & \text { R } & \text { E } & \text { K } & \text { S } & \text { V } & \text { W } & \text { I } & \text { L } & \text { I } & \text { T } & \text { V } & \text { F } & \text { C } & \text { A } & \text { F } & \text { I } & \text { F } & \text { L } & \text { I } & \text { V } & \text { T } & 250\end{array}$ TGG TTG CTA CAC ATG AAC AGC CGC AGT CTG AAG CAT TTT ATC CTG CCA CCA GTC CCT GGT CCT AAA ATC AAA GGA 825 $\begin{array}{llllllllllllllllllllllllll}\text { W } & \text { L } & \text { L } & \text { H } & \text { M } & \text { N } & \text { S } & \text { R } & \text { S } & \text { L } & \text { K } & \text { H } & \text { F } & \text { I } & \text { L } & \text { P } & \text { P } & \text { V } & \text { P } & \text { G } & \text { P } & \text { K } & \text { I } & \text { K } & \text { G } & 275\end{array}$ TTT GAT AAG CAG CTG CTC AAG AAC GGC AAG TCT GAG GAG GTC TTC AGT GCA CTG GTG GTG TCT GAC TTC CCC CCG 900 $\begin{array}{lllllllllllllllllllllllllllllll}F & D & K & Q & \text { H } & \text { L } & K & \text { N } & G & K & S & E & E & \text { V } & F & S & A & \text { L } & \text { V } & \text { V } & \text { S } & \text { D } & \text { F } & P & P & 300\end{array}$ AAG TCA TCT AC TAg GAG GAC TTG CTG GTG GAG TAC TTA GAA GTG TAT ATC CCC GAg GAg CAg GAg CTG ATG CTG 975

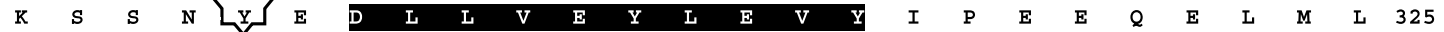
GAG AAA AGC ATG GAT CTG GAC GTC AGT TGC CTG AAA TCT GAG GGA TCC ACG TCC GAC AGC GAC TCT GGC CGg GGC 1050 $\begin{array}{lllllllllllllllllllllllllll}E & K & S & M & D & \text { L } & \text { D } & \text { V } & \text { S } & \text { C } & \text { L } & \text { K } & \text { S } & \text { E } & \text { G } & \text { S } & \text { T } & \text { S } & \text { D } & \text { S } & \text { D } & \text { S } & G & R & G & 350\end{array}$ AGC TGC GAC AGT CAT ACT CTG CTG ATG CAT AAG TGT GAT GGG GTA AAA GAA GGA AGA GAG AGg CAA GCG GAG GAG 1125 $\begin{array}{lllllllllllllllllllllllllllll}S & C & D & S & H & \text { T } & \text { L } & \text { L } & \text { M } & \text { H } & \text { K } & \text { C } & \text { D } & G & \text { V } & \text { K } & \text { E } & G & \text { R } & \text { E } & \text { R } & \text { Q } & \text { A } & \text { E } & E & 375\end{array}$ GCA CGG AGG CAT CAG AAG GAC TGG AAG GAA GAA GCC TCG ACC TÂ TCT AAT GTG GAC AGC AGC CCT GAC ATG TCC 1200 $\begin{array}{llllllllllllllllllllllllllll}A & R & R & H & Q & K & D & W & K & E & E & A & S & T & Y \\ Y & S & N & V & D & S & S & P & D & M & S & 400\end{array}$ AGT GGC AGG GTG AAG ACC TGG CCG TCT GTG TTT TCA CCG CTG CCC CGG TA9 AGC TCC AAC CAG CAG GGC TCG CTC 1275 $\left.\begin{array}{lllllllllllllllllllllllllll}S & G & R & V & K & T & W & P & S & V & F & S & P & \text { L } & P & R & Y\end{array}\right] S$ GAG ACG GCC AAA CAG CAC TAg CTC TCC GAC AGC CTT TTC CGC CCG AGC TCC ACG TCC TCC TGC CAC ACC CAG CCC 1350 $\begin{array}{llllllllllllllllllllllllll}\mathbf{E} & \mathrm{T} & \mathrm{A} & \mathrm{K} & \boldsymbol{Q} & \mathrm{H} & \mathrm{Y} & \mathrm{L} & \mathrm{S} & \mathrm{D} & \mathrm{S} & \mathrm{L} & \mathrm{F} & \mathrm{R} & \mathrm{P} & \mathrm{S} & \mathrm{S} & \mathrm{T} & \mathrm{S} & \mathrm{S} & \mathrm{C} & \mathrm{H} & \mathrm{T} & \mathrm{Q} & \mathrm{P} & 450\end{array}$ GGC CAC GGC GCC ACA GAG GCT CTC GGG CCG AGC PAC TGG GAG TGT GGC CTG AGC AAC AAg CAg CCT CAC CTG CTC 1425

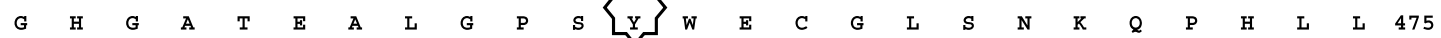
CAC CCC CAG GGG CAG GCT CAG AGG CAG CTC CAG GCC CAC AGC GAC GAC AAC ATC TCC AGC ATC GGC CGC AAG CCG 1500 $\begin{array}{llllllllllllllllllllllllllllll}H & P & Q & G & Q & A & \& & R & Q & L & Q & A & H & S & D & D & N & I & S & S & I & G & R & K & P & 500\end{array}$ ACG CCT GCC GGG CTG CCC TCG CCC GCT CTC CGG TCC ACC GAG TAC GTG GAG GTC CAG AGg GTC AAT GAG GAG GAT 1575

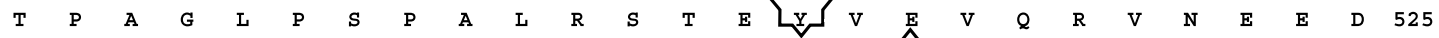
ATg GTG CTT CTC CAg CCT GTG TCA GGC CGA TGT GGC GAC GTG GAG GGC TAa CCC CAG GTG CCC CAC GGA GAG GAC 1650

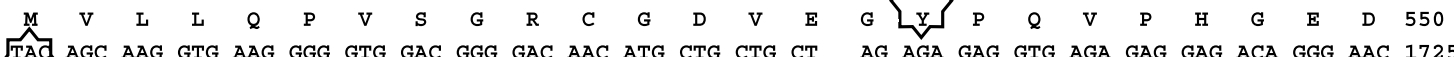

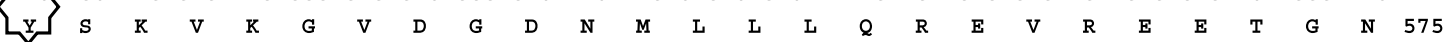
TGT GAC ACG CCG TCC ACA GTT GCC ACG ACA CAG AAG CCT ACA ACC TGC ATT CAC TCT GCC ATG CCG GTC CAG GGC 1800 $\begin{array}{lllllllllllllllllllllllllllllllllll}C & D & T & P & S & T & V & A & T & T & Q & K & P & T & T & C & I & H & S & A & M & P & V & Q & G & 600\end{array}$ GAA GCG GTC CTG GGA GTG AAC GGT TAO GTT GAC ACT GCC ACC GTT TTC ACA CTG CCT ACC FAC TAG 1866

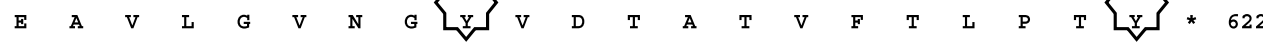
GTGCCCAAACTGGCAGGTGGG

Figure 1 Nucleotide and deduced aa sequences of the sbPRLR1. Nucleotides (upper sequence) and aa (lower sequence) are numbered on the right. The following features on the sequence are highlighted: conserved cysteine residues in the extracellular domain (circled), WS motif (underlined with a solid line), transmembrane domain (underlined with a dashed line), Box 1 and Box 2 regions (boxed in shaded rectangles), intracellular tyrosine residues (circled with polygons), and the stop codon (marked with an asterisk).

activation by sPRL could stimulate both the $\beta$-casein and c-fos promoters (Fig. 6A and B) but only the sbPRLR1 can stimulate the Spi 2.1 promoter (Fig. 6C). For the $\beta$-casein promoter, sbPRLR 1 could trigger a bigger stimulation than sbPRLR2. But for the c-fos promoter, the two receptors exhibited similar activities. This could not be attributed to the difference in the expression levels of the two receptors in the transfected cells since determination of receptor levels in the cultured cells by western blot (Fig. 6D) indicated that the two receptors had very similar expression levels in the transfected cells. Ligand specificity of receptor interaction was ascertained by stimulating the receptor-transfected cells with sGH and 
ACAGATGCGAGGGTTACCAGGTGTGAGAAGGTCGTCGACGACAATGTTGCGGCCGCAGG AGGATTCTGTTCGCTCTGTCGACAGCAGCAACATGGAGTTGAGTGACTCAGTGGTGTAGGTCGACACTCAGCTGGACACACTGCGGGACAGTTGGTGCC
ATG ATG TGG AGA GAT CTT CGA TTG GCA GTG CTT GTG CTG CTG TGT GCA GCT GTA AAG TCC AAC AGC TCG TCT CCA 75 $\begin{array}{lllllllllllllllllllllllllllll}M & M & \text { W } & R & \text { D } & \text { L } & R & \text { L } & \text { A } & \text { V } & \text { L } & \text { V } & \text { L } & \text { L } & \text { C } & \text { A } & \text { A } & \text { V } & \text { K } & \text { S } & \text { N } & \text { S } & \text { S } & S & P & 25\end{array}$ CCA GGG AAA CCA GTC CTG CTC AGC TGC AGG TCC CCT GAG AAG GAG ACG TTT ACG TGC TGG TGG CAg CCA GGC TCT 150

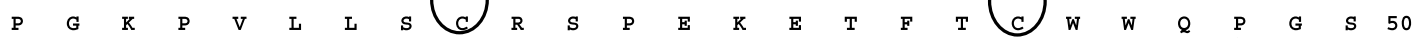
GAC GGA GGG CTG CCC ACC ACC TAC AAA CTC TAT TAT GAG AGA GAA AGA TTG GAG GGA GTG CAC GAG TGT CCG GAC 225 $\begin{array}{llllllllllllllllllllllllllllllllll}D & G & G & L & P & T & T & Y & K & L & Y & Y & E & R & E & R & L & E & G & V & H & E & C & P & D & 75\end{array}$ TAT CGG TCA GCA GGT AGC AAC TCC TGT TTC TTC AGC AAg AGC CAC ACC TCC ATC TGG GTT GAg tAC TAC CTG ACT 300 $\begin{array}{lllllllllllllllllllllllllll}\mathrm{Y} & \mathrm{R} & \mathrm{S} & \mathrm{A} & \mathrm{G} & \mathrm{S} & \mathrm{N} & \mathrm{S} & \mathrm{C} & \mathrm{F} & \mathrm{F} & \mathrm{S} & \mathrm{K} & \mathrm{S} & \mathrm{H} & \mathrm{T} & \mathrm{S} & \mathrm{I} & \mathrm{W} & \mathrm{V} & \mathrm{E} & \mathrm{Y} & \mathrm{Y} & \mathrm{L} & \mathrm{T} & 100\end{array}$ GTG GTG GCC TCC AAT GCC CTC GGg AAT GCC ACC TCA GAT GTC TTT AAA ATg GAT GTG ATg CAA ATA ATA AAg CCC 375 $\begin{array}{llllllllllllllllllllllllllll}\text { V } & \text { V } & \text { A } & \text { S } & \text { N } & \text { A } & \text { L } & \text { G } & \text { N } & \text { A } & \text { T } & \text { S } & \text { D } & \text { V } & \text { F } & \text { K } & \text { M } & \text { D } & \text { V } & \text { M } & \text { Q } & \text { I } & \text { I } & \text { K } & \text { P } & 125\end{array}$ AAT GTT CCT GAA AAT GTA ACG CTG CTG GTg GTG GAg ACG GGg GAC AGC CCA TAT CTC AAT ATC AGA TGG GAA CAT 450

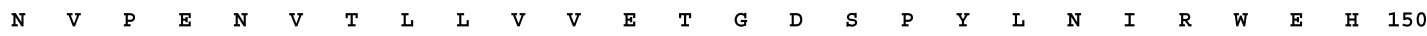
CCA CGT AAC ACA GAC ACC GGG TCC GGC TGG GTC ACC ATT AAA TAT GAA CTA AGA GTC AAA CAA GAA AAC AAT AAC 525 $\begin{array}{lllllllllllllllllllllllllll}\mathbf{P} & \mathrm{R} & \mathbf{N} & \mathrm{T} & \mathrm{D} & \mathrm{T} & \mathrm{G} & \mathrm{S} & \mathrm{G} & \mathrm{W} & \mathrm{V} & \mathrm{T} & \mathrm{I} & \mathrm{K} & \mathrm{Y} & \mathbf{E} & \mathrm{L} & \mathrm{R} & \mathrm{V} & \mathrm{K} & \mathbf{Q} & \mathbf{E} & \mathbf{N} & \mathbf{N} & \mathbf{N} & 175\end{array}$ AAG TGG AAG GCG TAC ATG TCA GGT ACA CAA ACC CAT TTC AGC CTG TAC AGC ATC ACT CCT GGA GTG GTG CAC ACA 600 $\begin{array}{llllllllllllllllllllllllllll}\text { K } & \text { W } & \text { K } & \text { A } & \text { Y } & \text { M } & \text { S } & \text { G } & \text { T } & \text { Q } & \text { T } & \text { H } & \text { F } & \text { S } & \text { L } & \text { Y } & \text { S } & \text { I } & \text { T } & \text { P } & \text { G } & \text { V } & \text { V } & \text { H } & \text { T } & 200\end{array}$ GTG CAG GTG CGC TGT AGA CTG GAC CAC GGC TCC TGG AGT GAC TGG ACC AAC AGT ACC CAC GTG AAG GTC CCA AAC 675 $\begin{array}{lllllllllllllllllllllllllllll}\mathrm{V} & Q & \mathrm{~V} & \mathrm{R} & \mathrm{C} & \mathrm{R} & \mathrm{L} & \mathrm{D} & \mathrm{H} & \mathrm{G} & \mathrm{S} & \mathrm{W} & \mathrm{S} & \mathrm{D} & \mathrm{W} & \mathrm{T} & \mathrm{N} & \mathrm{S} & \mathrm{T} & \mathrm{H} & \mathrm{V} & \mathrm{K} & \mathrm{V} & \mathrm{P} & \mathrm{N} & \mathbf{2} 25\end{array}$ TAT GTT CAG AAT GAg AAA TCA TTT TGG ATC TTG GTC GCC ACC CTG TCT GCA ATT CCA TTT ATg GCA GCA ATG TGC 750

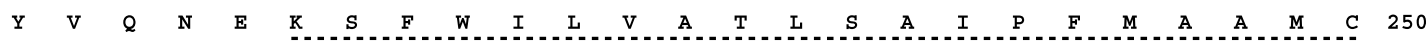
ATC TTG GTT GTA AAG AGG GAA AAT GTG AAG CAG TGT GTT CTG CCT CCT GTT CCT GGT CCA AAG ATC AGA GGA GTT 825 $\begin{array}{llllllllllllllllllllllllllll}I & \text { L } & \text { V } & \text { V } & \text { K } & R & \text { E } & \text { N } & \text { V } & \text { K } & Q & \text { C } & \text { V } & \text { L } & \text { P } & \text { P } & \text { V } & \text { P } & \text { G } & \text { P } & \text { K } & \text { I } & \text { R } & \text { G } & \text { V } & 275\end{array}$ GAT TTC CAC CTT CTC ATG AGT GGA CAA TAT GAA GAC ACC ACC AAT GCC CTG ATC ATC AAC CAG AAC TTT CCT CTC 900 $\begin{array}{llllllllllllllllllllllllllll}D & F & H & \text { L } & \text { L } & \text { M } & S & G & Q & \text { Y } & \text { E } & \text { D } & \text { T } & \text { T } & \text { N } & \text { A } & \text { L } & \text { I } & \text { I } & \text { N } & \text { Q } & \text { N } & \text { F } & \text { P } & \text { L } & 300\end{array}$ ATG ATG GGC TGG AAg AAC CAG ATC GAG GAG FAg CTC ATA GTG ACC GAG AAT AGC ATC GGG CCT CCG TTG GAT GCC 975 $\begin{array}{llllllllllllllllllllllllllll}M & M & G & \text { W } & \text { K } & \text { N } & \text { Q } & \text { I } & \text { E } & \text { E } & \text { Y } & \text { L } & \text { I } & \text { V } & \text { T } & \text { E } & \text { N } & \text { S } & \text { I } & \text { G } & \text { P } & \text { P } & \text { L } & \text { D } & \text { A } & 325\end{array}$ TCG AAT TGT CAA AAA AGG AAA AAg AGC TTG ATT ATT CCT GCT GGC TTC TGC TCA GAT TGG GAA ATt GAA TGC AAg 1050 $\begin{array}{llllllllllllllllllllllllllll}S & N & C & Q & K & R & K & K & S & \text { L } & I & I & P & A & G & F & C & S & D & \text { W } & E & I & E & C & K & 350\end{array}$ TCA ACC CAC ATT CAG AAT GAC TGT GCA GGG GGA ACA AAG AAT GAA ACA GGT AAC TTT CCC AAG AAC AAT AAG TCT 1125 $\begin{array}{llllllllllllllllllllllllllll}S & \text { T } & \text { H } & \text { I } & Q & \text { N } & \text { D } & \text { C } & \text { A } & \text { G } & \text { G } & \text { T } & \text { K } & \text { N } & \text { E } & \text { T } & \text { G } & \text { N } & \text { F } & \text { P } & \text { K } & \text { N } & \text { N } & \text { K } & \text { S } & 375\end{array}$

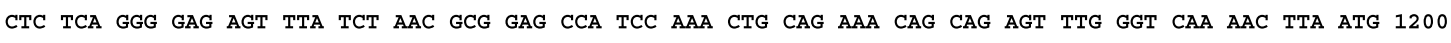
$\begin{array}{llllllllllllllllllllllllllll}\mathrm{L} & \mathrm{S} & \mathrm{G} & \mathrm{E} & \mathrm{S} & \mathrm{L} & \mathrm{S} & \mathrm{N} & \mathrm{A} & \mathrm{E} & \mathrm{P} & \mathrm{S} & \mathrm{K} & \mathrm{L} & \& & \mathrm{~K} & \& & \& & \mathrm{~S} & \mathrm{~L} & \mathrm{G} & \& & \mathrm{~N} & \mathrm{~L} & \mathrm{M} & 400\end{array}$ AAC ACA GAA GCG ACA GAA CCC AGT CCA CTG AAC CAG GAG AAC GAC GTC AAA CTC TTC ACA AAC AGC GAC TAC GTG 1275 $\begin{array}{lllllllllllllllllllllllllll}\mathbf{N} & \mathrm{T} & \mathrm{E} & \mathrm{A} & \mathrm{T} & \mathrm{E} & \mathrm{P} & \mathrm{S} & \mathrm{P} & \mathrm{L} & \mathrm{N} & \boldsymbol{Q} & \mathrm{E} & \mathrm{N} & \mathrm{D} & \mathrm{V} & \mathrm{K} & \mathrm{L} & \mathrm{F} & \mathrm{T} & \mathrm{N} & \mathrm{S} & \mathrm{D} & \mathrm{V} & \mathrm{V} & \mathbf{4 2 5}\end{array}$ GAT ATT CGg AAA CAT GTG GAG AAg ACG CAg GAG GTG AAT GTG AAA CAG TTG GAC TAa AGC AGA ATg GAG GAA GTG 1350

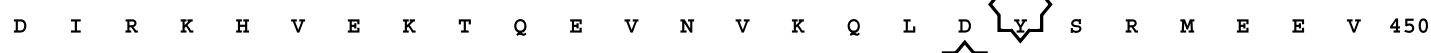
AAT GGT GAC AAT GTG CTC ATC CTC AAC AAC AAA AAT CTC CCC TTT AAC AGC TAO ATG GAT TTt CAg AGg CAA GAG 1425

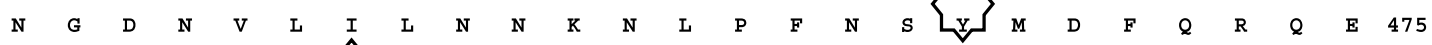
GAG AAA CTA TCA GCG GAT TAO AGC AGG GTG AAA GAG GTG GAC AGT AAC AAT GTA GTG GTC CTG CAG AGG CAA GAC 1500

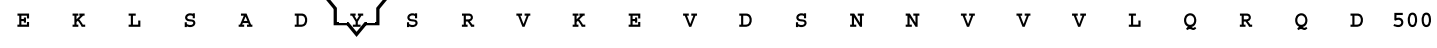
GTG TCA GCT GAC ACT AAA ACT CTT CAT GCG ACT GGA GCC CAC AAA ATG GGG GTG TGC ACA GAA CTC ATC AAC AGC 1575 $\begin{array}{llllllllllllllllllllllllll}\mathrm{V} & \text { S } & \text { A } & \text { D } & \text { T } & \text { K } & \text { T } & \text { L } & \text { H } & \text { A } & \text { T } & \text { G } & \text { A } & \text { H } & \text { K } & \text { M } & \text { G } & \text { V } & \text { C } & \text { T } & \text { E } & \text { L } & \text { I } & \text { N } & \text { S } & 525\end{array}$ GGA IÂT GTA GAT ACC ATC CCT GCA TCA CCT TTA ATG TAA 1613

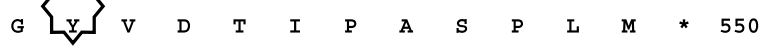

TGTTTTAACATGCAATGTCCATATACAAATGGTAGATGTCCCTTCTGATTTACTGTTCAGAGTTCAGAGAAAAAGGCTGATGGTATTGTGTAGTTTTGTCAATAT AATCTGAGGAGTTTTCCATGACATTTGGCACAGACATTCATGGTCTCCAGAAGATAAATCTTACCAACTTCAGTGACACCCTGAACTTCCCTATAGCATCATGAC GTTGCTGCTTTTGTTTCAGAATGAAATATCTTGACACTTTGGAATTTATTGTTGTGAAATTTGATACATATATTCAAGGTCCCCAGAGAATAAACCTAAAATCTT TGGTGATCCCGACTCTCTATCTAGCCCCATAATCATCCCAAAGTTTTAATATATCACCTGACTTACTGTATGTAACAGTAGTTGTAGTTGTAATTATTACATGAT AGGTATAAGGATATACGTTTTATGGCTCAGGCTAAGGTAACTTTTTAGAAATCTGAAAAGAAAAATACTCAGCCACTCCTTCTTAGATGCTTGCCACTACTGGGA CACCTGGTGCATTGTACAGTGTATTGATGCATTGAAGCACAGGTCATGTCTAGTCTCCAAGCTATACTACCTTGTGGTGGAAGTTACCAGTTGCCCTTTAAAGAA AATCCTGCACTGTATTTCACCAGTCTGACTGAAGTTTTTTCTCCAGGTGGTGTGAAGTAAAACCATATGATAACATGATTACTCTCAGACAGGACTATTTTTACT CCTCGTGAAACTGTCGATTACTTATTCTGAATTTGAGTACATCATTTCTTAATTCCAATCCGATATATTTTGGAGGAAAATACTTTTTTACTCTACTGTACTTTT TCAGTTGTTGAGGAAATACTCACTTTGGTAACAAAGTGATACCACAGTGTAAAAATACATGTATGCATAAACGTCCTATTCATCATAATTATTTATGTATTAAAC GATAAGTTTCACCCAAATTGAAAAAAAAAAAAAA

Figure 2 Nucleotide and deduced aa sequences of the sbPRLR2. Nucleotides (upper sequence) and aa (lower sequence) are numbered on the right. The conserved cysteine residues in the extracellular domain, WS motif, transmembrane domain, Box 1 region, intracellular tyrosine residues, and the stop codon are highlighted in the same way as in Fig. 1. 
CAGTGGTCGCCAGGTGTGCGGAGCTC GGCTGGAGCTCGTTTGGCTTTGTGTCATGACTGAAGAAGGCTACCTTTTTTGTTCTGTACCCGTCAACATCCGATAAATATCGATGATAAACATAG CTGCCAAGATCATAAAGTGGTAACAACAAGACACTCGAGCTCGGATCACACCATTACCACTGGCTTGTGATGTCACTTCCCTGCCACAGAGTGTG ATG GTT TGT GCC AGG ATG GTG AAg CAT CTC AGG CTG CCA TTG CTG CTC CTG CTG CTG GCA TTA GCT GCA GAA TGO 75 $\begin{array}{lllllllllllllllllllllllllll}M & V & C & A & R & M & V & K & \text { H } & \text { L } & R & \text { L } & \text { P } & \text { L } & \text { L } & \text { L } & \text { L } & \text { L } & \text { L } & \text { A } & \text { L } & \text { A } & \text { A } & E & \text { C } & 25\end{array}$ AAC AGC ATG TCT AAA CCA GGA AAA CCA GAC AAA CTT GGC TGC AGg TCC CCC GAC AAg GAg ACT TTt TTC TGC TGG 150

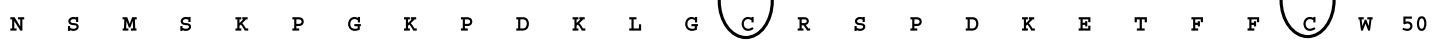
TGG ACG CCA GGC TCT GGT GGA GGG CTG CCC ACT GTG CAT CGC CTC TAC TAT AAG ACA GAA GGT AGg GAG ACT GTA 225 $\begin{array}{lllllllllllllllllllllllllllll}\text { W } & T & \text { P } & G & S & G & G & G & \text { L } & P & \text { T } & \text { V } & \text { H } & \text { R } & \text { L } & \text { Y } & \text { Y } & \text { K } & \text { T } & \text { E } & \text { G } & \text { R } & \text { E } & \text { T } & \text { V } & 75\end{array}$

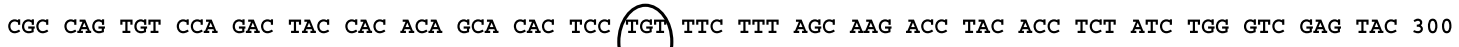
$\begin{array}{lllllllllllllllllllllllllllll}R & Q & C & P & D & Y & H & T & A & H & S & C & F & F & S & K & T & Y & T & S & I & W & V & E & Y & 100\end{array}$ ACT GTG ATT GTG GAG GCC TCC AAT GCC CTT GGG AAT ACA TCT TCA GAC CCC CTT ACT TTT GAC TTG ATG GAC ATT 375 $\begin{array}{llllllllllllllllllllllllllll}\text { T } & \text { V } & \text { I } & \text { V } & \text { E } & \text { A } & \text { S } & \text { N } & \text { A } & \text { L } & \text { G } & \text { N } & \text { T } & \text { S } & \text { S } & \text { D } & \text { P } & \text { L } & \text { T } & \text { F } & \text { D } & \text { L } & \text { M } & \text { D } & \text { I } & 125\end{array}$ GTG AAG CCC TAT GCA CCT GAA AAC GTT ACA CTG GTG GTC AGC ATA GGG GAC AAT CCA CAC CTC ACC ATC CAA TGG 450 $\begin{array}{llllllllllllllllllllllllllll}\mathrm{V} & \mathrm{K} & \mathrm{P} & \mathrm{Y} & \mathrm{A} & \mathrm{P} & \mathrm{E} & \mathrm{N} & \mathrm{V} & \mathrm{T} & \mathrm{L} & \mathrm{V} & \mathrm{V} & \mathrm{S} & \mathrm{I} & \mathrm{G} & \mathrm{D} & \mathrm{N} & \mathrm{P} & \mathrm{H} & \mathrm{L} & \mathrm{T} & \mathrm{I} & \mathrm{Q} & \mathrm{W} & 150\end{array}$ CAC ACA CCG TCT AAC ATA GAC ACA AAG TCA GGA TGG GTC ACT CTG AAG AAT CAA CTG AGA ATC AAA CTA GAA AAG 525 $\begin{array}{llllllllllllllllllllllllllll}\text { H } & \text { T } & \text { P } & \text { S } & \text { N } & \text { I } & \text { D } & \text { T } & \text { K } & \text { S } & \text { G } & \text { W } & \text { V } & \text { T } & \text { L } & \text { K } & \text { N } & \text { \& } & \text { L } & \text { R } & \text { I } & \text { K } & \text { L } & \text { E } & \text { K } & 175\end{array}$ AGC AAA ATA TGG AAg AAT tAC ACG TCA GAT ACA CAA ACA CAT TTT ACC ATT TAC AAC ATt GAA CTT GGG GTG GTG 600 $\begin{array}{llllllllllllllllllllllllllllll}S & K & I & W & K & N & Y & T & S & D & T & Q & T & H & F & T & I & Y & \text { N } & \text { I } & \text { E } & \text { L } & \text { G } & \text { V } & \text { V } & 200\end{array}$ TAC ATG GTC GAA GTG CGC TGT GCA ATA GAC AAT AGC GCC TGG AGT GAG TGG ACC AAC ACT ACC TTT GTC AAA GTA 675 $\begin{array}{lllllllllllllllllllllllllllll}\mathbf{Y} & \mathrm{M} & \mathrm{V} & \mathrm{E} & \mathrm{V} & \mathrm{R} & \mathrm{C} & \mathrm{A} & \mathrm{I} & \mathrm{D} & \mathrm{N} & \mathrm{S} & \mathrm{A} & \mathrm{W} & \mathrm{S} & \mathrm{E} & \mathrm{W} & \mathrm{T} & \mathrm{N} & \mathrm{T} & \mathrm{T} & \mathrm{F} & \mathrm{V} & \mathrm{K} & \mathrm{V} & \mathbf{2} 25\end{array}$ CCT AAC TTT CCT CGC ACG GAC AAT TCC TTT TGG ATA CTG GTT TTC AGC CTC TCT CTG ATT CCG TTG CTT GTA GCA 750

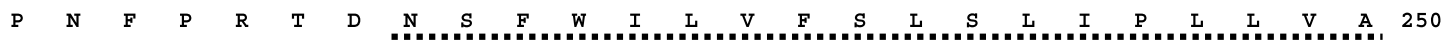
ATG ATT ATC TTG ATC CTG AAG AGA AAA ACT GTG AAG AAG TAT ATT CTG CCC CCT GTT CCT GGT CCA AAG ATA AGA 825

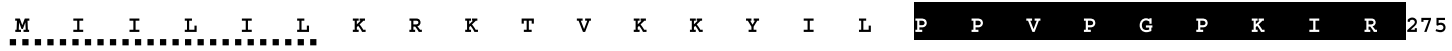
GGA GTT GAT GTT CAA CTT CTC AAG AGC GGC CGA TCT GAA GAT GTC ATT AAT GCC ATG CTT CTC AAC CAG AGA TTT 900 $\begin{array}{llllllllllllllllllllllllllll}G & V & D & V & Q & L & L & K & S & G & R & S & E & D & V & I & N & A & M & L & L & N & Q & R & F & 300\end{array}$ CCT GTT GTG ATg CCC TGG AAg GAC CAG AAT GAG GAT TAg CTG ATC GTG TCT GAT AAA GAA ACC TCG TAM TTG CCA 975

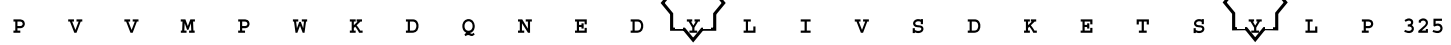
TTG GAA AAA AAT AAA ATA TTC ATT CTG CCA GTT GCC ATA ACA TTA GAC TCA GAA GTC CAA TCT AAg GAG ACA AGT 1050 $\begin{array}{lllllllllllllllllllllllllllll}\mathrm{L} & \mathrm{E} & \mathrm{K} & \mathrm{N} & \mathrm{K} & \mathrm{I} & \mathrm{F} & \mathrm{I} & \mathrm{L} & \mathrm{P} & \mathrm{V} & \mathrm{A} & \mathrm{I} & \mathrm{T} & \mathrm{L} & \mathrm{D} & \mathrm{S} & \mathrm{E} & \mathrm{V} & \mathrm{Q} & \mathrm{S} & \mathrm{K} & \mathrm{E} & \mathrm{T} & \mathrm{S} & 350\end{array}$ GAA GTG GAT CGC TTT CTA AAA GAC AGT GAg TCT TCC TCA GAG GAg Agt TCA GAg AAg ACC AAg TCG TCG CAG CTT 1125 $\begin{array}{llllllllllllllllllllllllllll}E & V & D & R & F & \text { L } & K & \text { D } & S & E & S & S & S & E & E & S & S & E & K & \text { T } & \text { K } & S & S & Q & \text { L } & 375\end{array}$ CTT ACT AAA TGC CCA AGT ACA AAC GTT TTG AAT GAT GAG AAA GCA AAT CAG CTA AAA GGT GTT ACT GAG AAG ATC 1200 $\begin{array}{lllllllllllllllllllllllllll}\mathrm{L} & \mathrm{T} & \mathrm{K} & \mathrm{C} & \mathrm{P} & \mathrm{S} & \mathrm{T} & \mathrm{N} & \mathrm{V} & \mathrm{L} & \mathrm{N} & \mathrm{D} & \mathrm{E} & \mathrm{K} & \mathrm{A} & \mathrm{N} & \mathrm{Q} & \mathrm{L} & \mathrm{K} & \mathrm{G} & \mathrm{V} & \mathrm{T} & \mathrm{E} & \mathrm{K} & \mathrm{I} & 400\end{array}$ ATC CAA CCC TTT GGA ATC AGC GGT TAT GTG GAT ATT CCA AGA CAT GAG CAC ACA CAG GAA GCA GAC TAC AGC CGA 1275

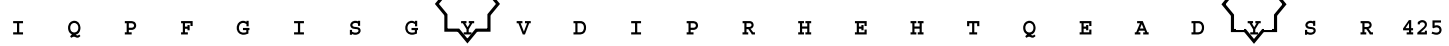
GTG AAC GAG GTG AAC AGT GAC AAT ATT TTA ATC ACG AAG AAA GAA AAT GTT CCC CAT ACT CTG GAC ACA CAG GGG 1350 $\begin{array}{llllllllllllllllllllllllllllll}\mathrm{V} & \mathbf{N} & \mathbf{E} & \mathrm{V} & \mathbf{N} & \mathrm{S} & \mathrm{D} & \mathbf{N} & \mathrm{I} & \mathrm{L} & \mathrm{I} & \mathrm{T} & \mathrm{K} & \mathrm{K} & \mathrm{E} & \mathrm{N} & \mathrm{V} & \mathrm{P} & \mathrm{H} & \mathrm{T} & \mathrm{L} & \mathrm{D} & \mathrm{T} & \mathrm{Q} & \mathrm{G} & 450\end{array}$ CAA GAA GGC GGT GTG CTC GAT GAC TAg AGC AGG GTG AAA GAA GTG AAT AGT GAC AAC ACT GTT TTC CTG GAG AAA 1425

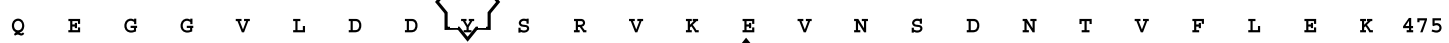
CAC AGT GAC TCA GTT AAT GCT TCT ATT AGg GCT AAA GGC TAg ATA GAC TGG GTC AAT CAg AAg GCA AAA AGT CC 1500 $\begin{array}{llllllllllllllllllllllllllllll}\text { H } & S & D & S & V & N & A & S & I & R & A & K & G & Y & I & D & \text { W } & \text { V } & \text { N } & \text { Q } & \text { K } & \text { A } & K & S & P & 500\end{array}$ CAT GTG ACT GGG CAC AGT GAA ATG GGA GTG TGC CCA CAA CTA GTT GGT TGT GGA FAT GTA GAT ACT TTC CCC CCA 1575

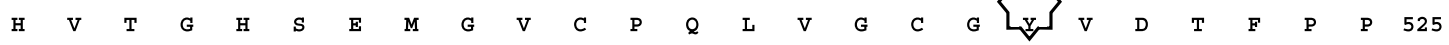
CCA GCT GTA ATC TAA 1590

$P \quad A \quad V \quad I \quad * 550$

AGCTTTTTATCATATAAAAATGCTATTATAAGGTGACAGCGGACATTCAGCATTACTTACTGTTGAATCAAAGCTGTCAATATTCTATATTTTTGCTGTTC TCGTTGGATGTATGGTTGGTACAGACATTTGTAAAACCATAAGATGTGCCATCTCTGGTCCAGAGTAAAATTTTATGACAGCTACGGAAAAAAAAAAAAAA

Figure 3 Nucleotide and deduced aa sequences of the ntPRLR2. Nucleotides (upper sequence) and aa (lower sequence) are numbered on the right. The conserved cysteine residues in the extracellular domain, WS motif, transmembrane domain, Box 1 region, intracellular tyrosine residues, and the stop codon are highlighted in the same way as in Fig. 1.

sSL in parallel. It was found that $\mathrm{sGH}$ and sSL could not stimulate the $\beta$-casein, $c$-fos, or Spi 2.1 promoters at a concentration where stimulation by SPRL could be readily demonstrated (Fig. 6). It is worth noting that for most of the positive response curves in Fig. $6 \mathrm{~A}-\mathrm{C}$, a characteristic bell-shaped dose-response relationship was found, indicating the requirement of a certain ratio between the ligand and the receptor in order to generate a maximal productive biological 


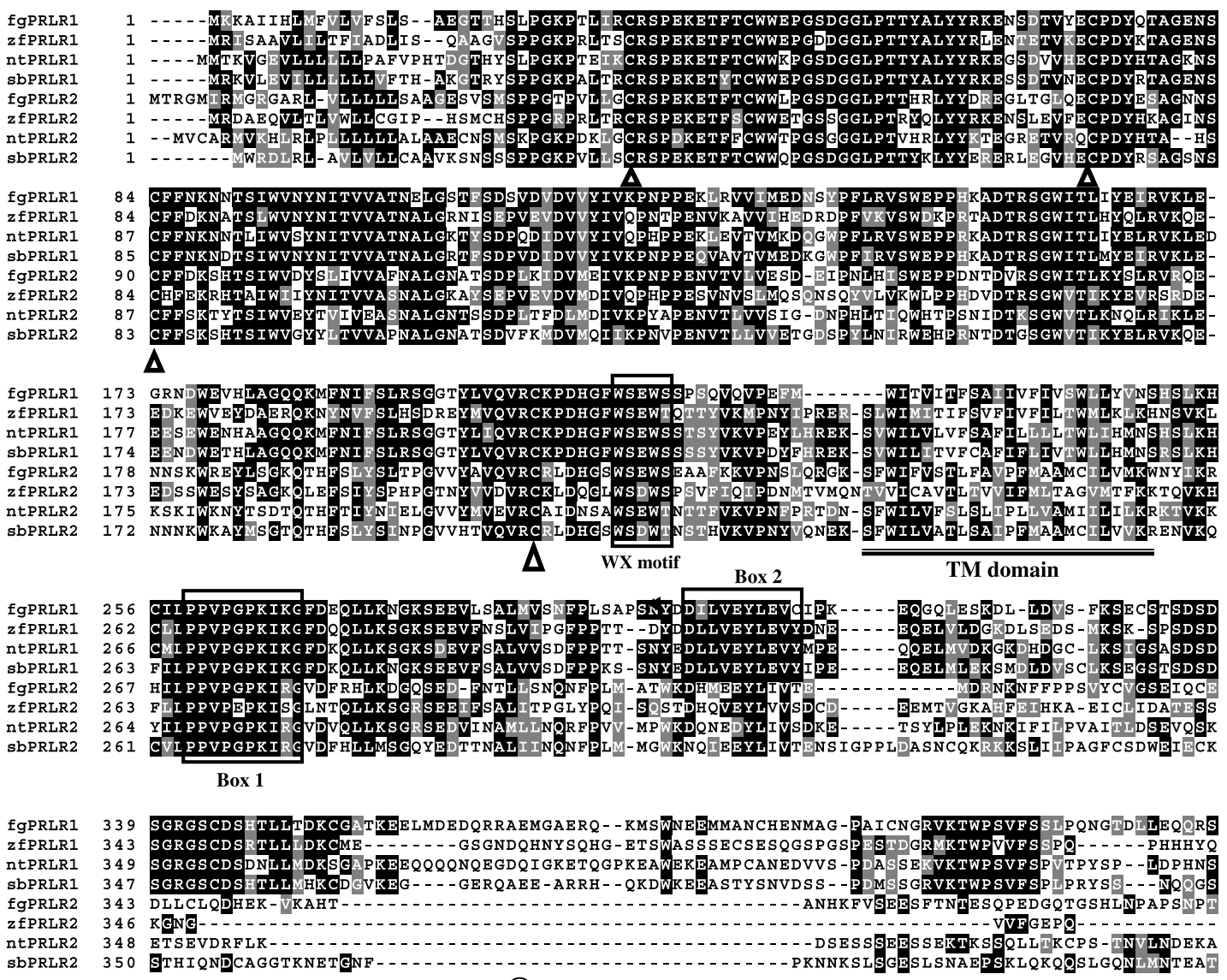

(1)

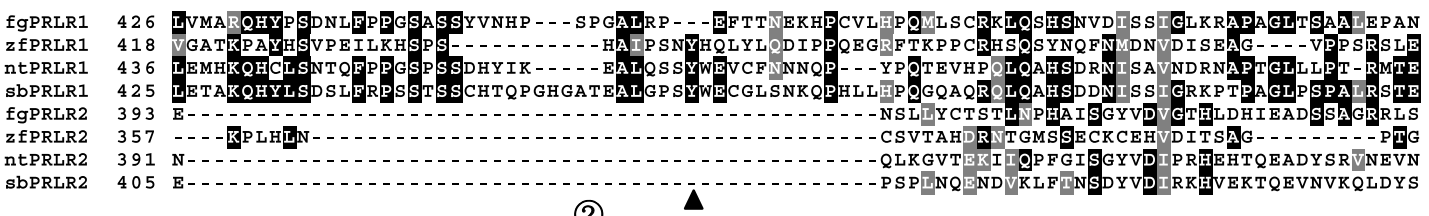

(2)

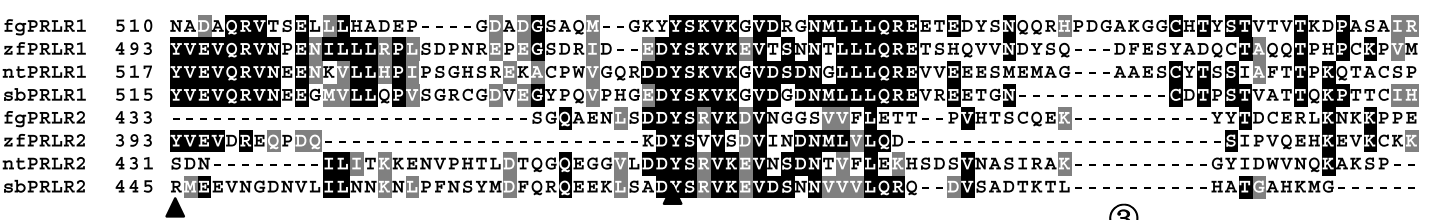
$\mathrm{R}$

(3)

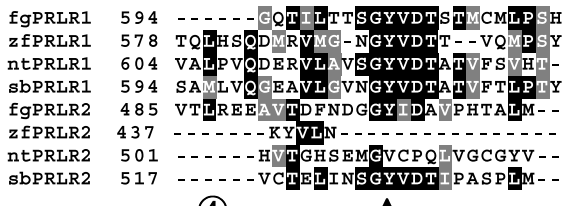

(4)

$\Delta$

Figure 4 Multiple alignment of PRLR1 and PRLR2 in fish where both sequences are known to date. These polypeptide sequences were aligned by the Clustal X program. The conserved cysteine residues in the extracellular domain are marked with white triangles at the bottom of the aligned sequences. The WS motifs are boxed. The transmembrane (TM) domains are underlined with two solid lines. The intracellular Box 1 and Box 2 regions are also boxed. The intracellular conserved tyrosine residues are marked with black triangles at the bottom of the aligned sequences. The major deleted regions in the intracellular domain of PRLR2 are numbered at the bottom of the aligned sequences. The abbreviations $\mathrm{fg}$, $\mathrm{zf}, \mathrm{nt}$, and sb stand for fugu, zebrafish, Nile tilapia, and black seabream respectively. 
Table 2 Amino acid similarity between fish prolactin receptors 1 (PRLR1s) and PRLR2s

\begin{tabular}{|c|c|c|c|c|}
\hline & PRLR1 & & & \\
\hline & B_seabream & N_tilapia & Fugu & Zebrafish \\
\hline PRLR2 & & & & \\
\hline G_seabream & $32 \cdot 9$ & $32 \cdot 9$ & $32 \cdot 0$ & $34 \cdot 4$ \\
\hline B_seabream & $32 \cdot 5$ & $32 \cdot 1$ & $31 \cdot 3$ & $32 \cdot 1$ \\
\hline N_tilapia & $31 \cdot 0$ & $29 \cdot 2$ & $27 \cdot 9$ & $30 \cdot 6$ \\
\hline Fugu & $34 \cdot 9$ & $32 \cdot 5$ & $32 \cdot 0$ & $32 \cdot 0$ \\
\hline Zebrafish & $39 \cdot 5$ & $36 \cdot 5$ & $36 \cdot 3$ & $35 \cdot 8$ \\
\hline
\end{tabular}

The Clustal method of the DNASTAR software was employed to compare PRLR1 and PRLR2 polypeptide sequences among the fish. Data are expressed as percentage aa identity. $G_{-}$seabream and B_seabream stand for gilthead seabream and black seabream respectively. N_tilapia stands for Nile tilapia.

response (Tse et al. 2000) due to the one ligand-two receptors proportionality. It is apparent that this proportionality would give rise to different dose-response curves for different receptors and for different signaling events.

\section{Tissue distribution}

Real-time PCR methods were developed to detect the transcript levels of sbPRLR1 and sbPRLR2 in different seabream tissues. The receptors appeared to be highly expressed in the osmoregulatory organs, particularly the gill and the intestine. In intestine and kidney, sbPRLR1 has a higher expression than sbPRLR2. But in the gill, the expression of sbPRLR2 is much higher than that of sbPRLR1. The expression level is similar for both receptors in the gonad. The expression levels of both receptors are relatively low in most other tissues, with sbPRLR 1 generally higher than sbPRLR2. The expression of sbPRLR2 in the heart and spleen could not be detected even using this sensitive real-time PCR method (Fig. 7).

\section{Differential regulation of sbPRLR 1 and $s b P R L R 2$ expression by steroid hormones in seabream kidney in vivo}

The expression of sbPRLR1 and sbPRLR2 in the kidney of seabream administered with different steroid hormones in vivo was investigated (Fig. 8). The expression of sbPRLR1 was significantly increased after treatment with $\mathrm{E}_{2}$ and cortisol, but treatment with testosterone did not elicit any significant change. In contrast, expression of sbPRLR 2 was significantly decreased after treatment with $\mathrm{E}_{2}$ and testosterone, while treatment with cortisol did not bring about any significant change (Fig. 8).

\section{Isolation of the $5^{\prime}$-flanking regions of the SbPRLR1 and SbPRLR2 genes}

As a first step towards understanding the transcriptional regulation of the seabream PRLR1 and PRLR2 genes, the $5^{\prime}-$ flanking regions of both genes were isolated in the present study. After several rounds of genome walking in a stepwise manner, two genomic fragments of 2366 and $2113 \mathrm{bp}$ corresponding to the $s b P R L R 1$ and $s b P R L R 2$ genes respectively were isolated. Sequence analysis using TFSEARCH (http://www.cbrc.jp/ research/db/TFSEARCH.html) and TESS (http://www.cbil. upenn.edu/cgi-bin/tess/tess?RQ = SEA-FR-QueryS)

revealed a number of putative transcription factor-binding sites on them (Figs 9 and 10). The promoter activities of these $5^{\prime}$ flanking regions were evaluated in cultured GAKS cells by measuring the promoter-driven luciferase activities. A 25-fold increase in luciferase activity over and above the promoter-less control (pGL3-Basic) was recorded for the sbPRLR1 gene promoter, and a 10-fold increase for the sbPRLR2 gene promoter (Fig. 11A). These strong promoter activities indicated that the cloned $5^{\prime}$-flanking regions did represent the functional promoters of the seabream PRLR genes.

\section{Regulation of promoter activities by steroid hormones}

As shown in Fig. 11, after treatment with $\mathrm{E}_{2}$ and cortisol, the luciferase activity was significantly increased for the $s b P R L R 1$ gene promoter, but decreased for the $s b P R L R 2$ gene promoter. In the case of testosterone treatment, the luciferase activity was significantly decreased for the $s b P R L R 2$ gene promoter, but remained unchanged for the $s b P R L R 1$ gene promoter. The overall promoter study results in response to steroid hormone challenge were in general agreement with the hormonal regulation of gene expression studies in vivo shown in Fig. 8.

Table 3 Amino acid sequence similarity among the fish prolactin receptors 2 (PRLR2s)

\section{PRLR2}

\begin{tabular}{|c|c|c|c|c|}
\hline G_seabream & B_seabream & N_tilapia & Fugu & Zebrafish \\
\hline \multicolumn{5}{|l|}{100} \\
\hline $86 \cdot 9$ & 100 & & & \\
\hline $41 \cdot 0$ & $41 \cdot 0$ & 100 & & \\
\hline $46 \cdot 3$ & $45 \cdot 9$ & $37 \cdot 6$ & 100 & \\
\hline $35 \cdot 6$ & $34 \cdot 0$ & $29 \cdot 7$ & $34 \cdot 5$ & 100 \\
\hline
\end{tabular}

The Clustal method of the DNASTAR software was employed to compare PRLR2 polypeptide sequences among the fish. Data are expressed as percentage aa identity. G_seabream and B_seabream stand for gilthead seabream and black seabream respectively. N_tilapia stands for Nile tilapia. 


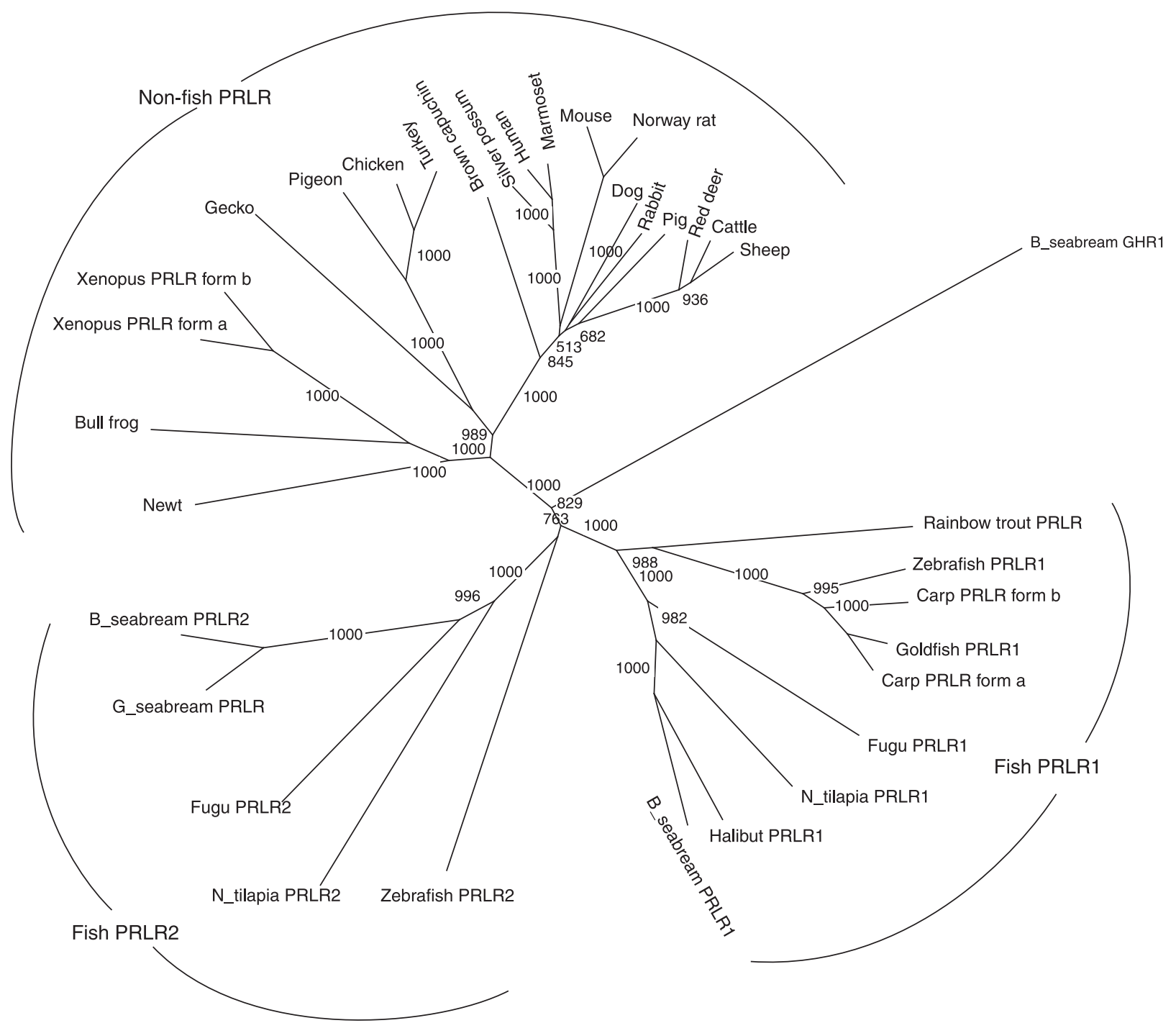

Figure 5 Phylogenetic tree of PRLRs from different vertebrates. The default settings of the ClustalX protein alignment program was employed using the black seabream GHR1 (NCBI accession no. AF502071) as the outgroup, and visualized by the treeview program. The values represent bootstrap scores out of 1000 trials, indicating the credibility of each branch. The two fish PRLR clades are named as PRLR1 and PRLR2. The PRLR sequences used in this phylogenetic analysis are: Bull frog (Rana catesbeiana) BAD14941; African clawed frog (Xenopus laevis) PRLR form a AAF05776 and form b AAF05777; Japanese firebelly newt (Cynops pyrrhogaster) BAB61107; silver-gray brushtail possum (Trichosurus vulpecula) AAD27039; brown capuchin (Cebus apella) AAO73437; dog (Canis familiaris) XP_536502; domestic pigeon (Columba livia) Q90374; rabbit (Oryctolagus cuniculus) P14787; chicken (Gallus gallus) AAS93635; pig (Sus scrofa) AAQ76842; human (Homo sapiens) AAH59392; turkey (Meleagris gallopavo) AAB01544; house mouse (Mus musculus) Q08501; Norway rat (Rattus norvegicus) P05710; red deer (Cervus elaphus) CAA64419; white-tufted-ear marmoset (Callithrix jacchus) CAB75847; cattle (Bos taurus) Q28172; sheep (Ovis aries) O46561; leopard gecko (Eublepharis macularius) BAD24103; zebrafish (Danio rerio) PRLR1 AAQ84555 and PRLR2 XP_690339; goldfish (Carassius auratus) AAF23268; common carp (Cyprinus carpio) PRLR form a AAK95833 and form b AAV71059; rainbow trout (Oncorhynchus mykiss) AAG44267; the Nile tilapia (Oreochromis niloticus) PRLR1 Q91513; bastard halibut (Paralichthys olivaceus) BAB62756; gilthead seabream (Sparus aurata) AAG17629; fugu (Takifugu rubripes) PRLR1 BAE94535; and PRLR2 NM_001078625; together with the three sequences obtained in the present study: black seabream (Acanthopagrus schlegeli) PRLR1 EF429092 and PRLR2 EF429093; and the Nile tilapia (Oreochromis niloticus) PRLR2 EF429094. 
A

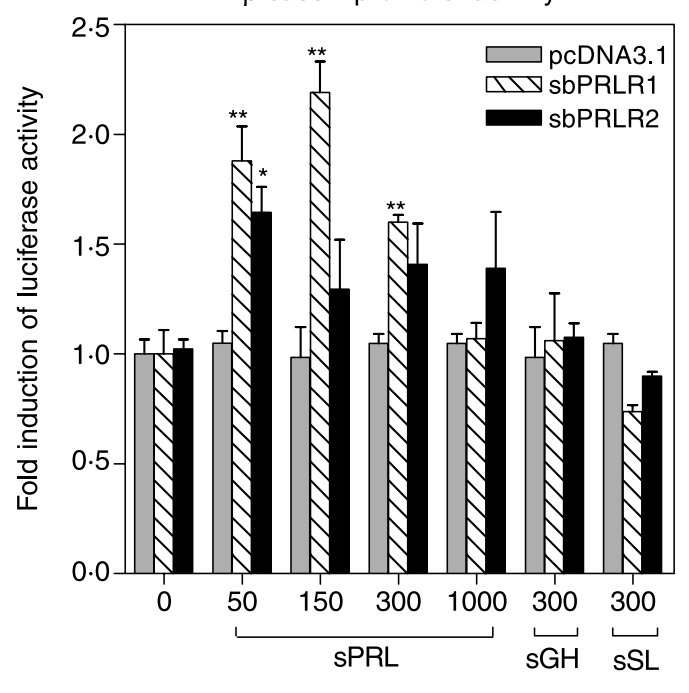

Concentrations of hormones $(\mathrm{ng} / \mathrm{ml})$

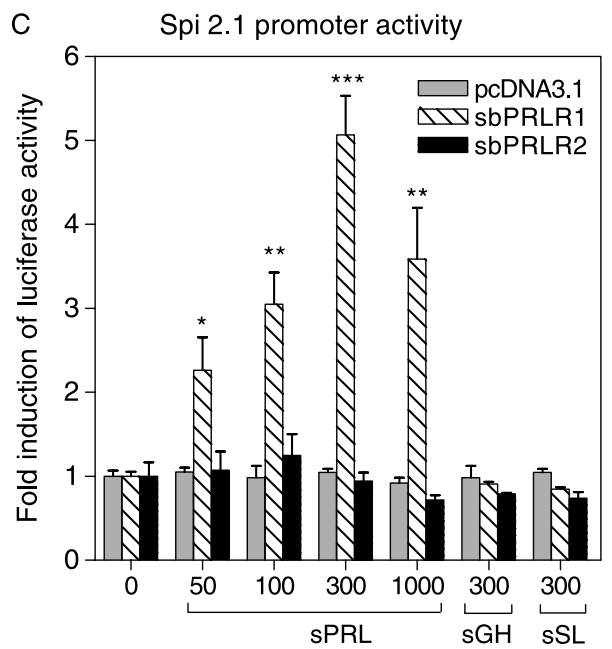

Concentrations of hormones $(\mathrm{ng} / \mathrm{ml})$

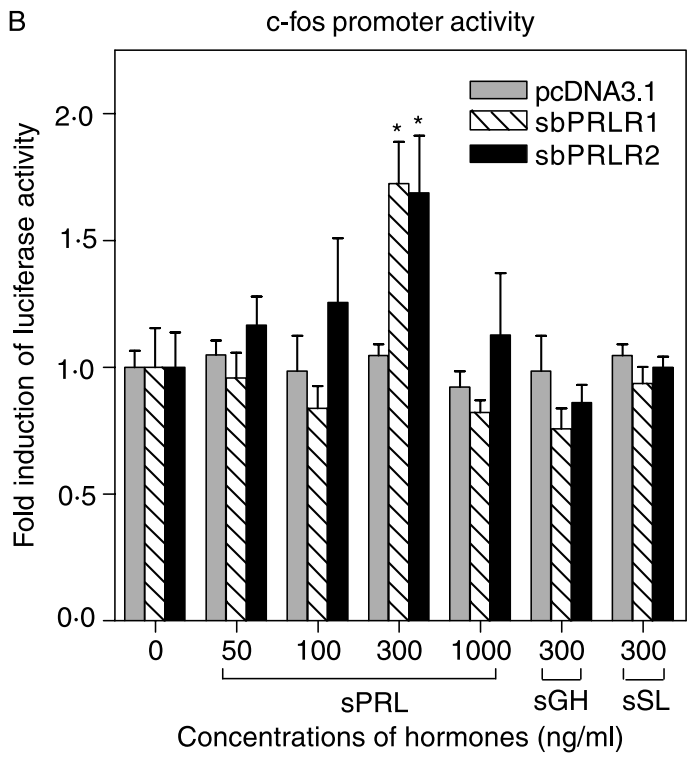

D

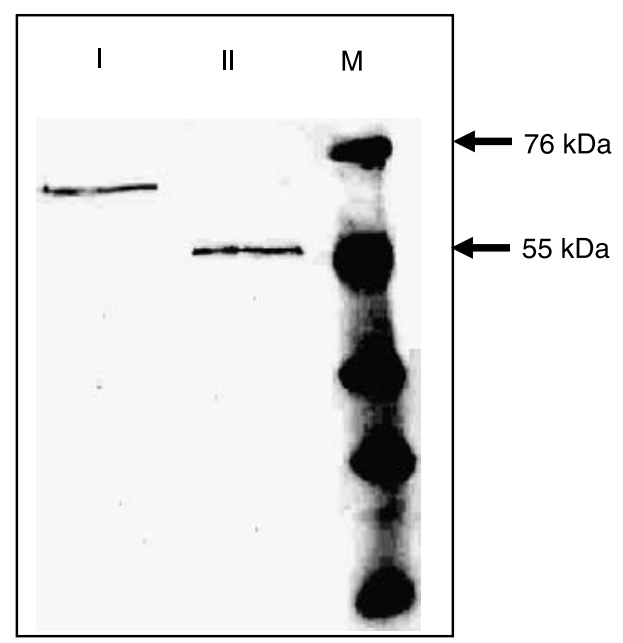

Figure 6 Transactivation of the different promoters in transfected HEK293 cells expressing sbPRLR1 and sbPRLR2. HEK293 cells were co-transfected with a pcDNA3.1 vector containing the entire coding region of either sbPRLR1 or sbPRLR2 (both containing a c-myc tag attached to the $C$ terminus of the receptor) together with a luciferase reporter plasmid driven by the $\beta$-casein promoter ( $A$ ), the $c$-fos promoter (B), or the Spi 2.1 promoter (C). The control for the receptor constructs was the empty pcDNA3.1 vector. The transfected cells were subsequently stimulated by salmon PRL (sPRL) at concentrations indicated on the $x$-axis, salmon GH (sGH) and salmon SL (sSL) at the concentration of $300 \mathrm{ng} / \mathrm{ml}$. Results are mean values \pm s.E.m. $(n=6 ; * P<0 \cdot 05 ; * * P<0 \cdot 01 ; * * * P<0 \cdot 001$ as compared with the respective control by one-way ANOVA). The western blot result by the anti-c-myc antibody is shown in (D). I and II represent the western blot results of sbPRLR1-myc and sbPRLR2-myc, at the appropriate sizes of the about 68 and 56 kDa respectively. No other major bands could be seen. $M$ represents the molecular weight standards. The results show that the two constructs had similar transfection efficiency and the expression levels of the two receptors were similar in the transfected cells. Cells transfected with the empty vector did not exhibit any of the receptor bands when western blotted with the anti c-myc antibody. 


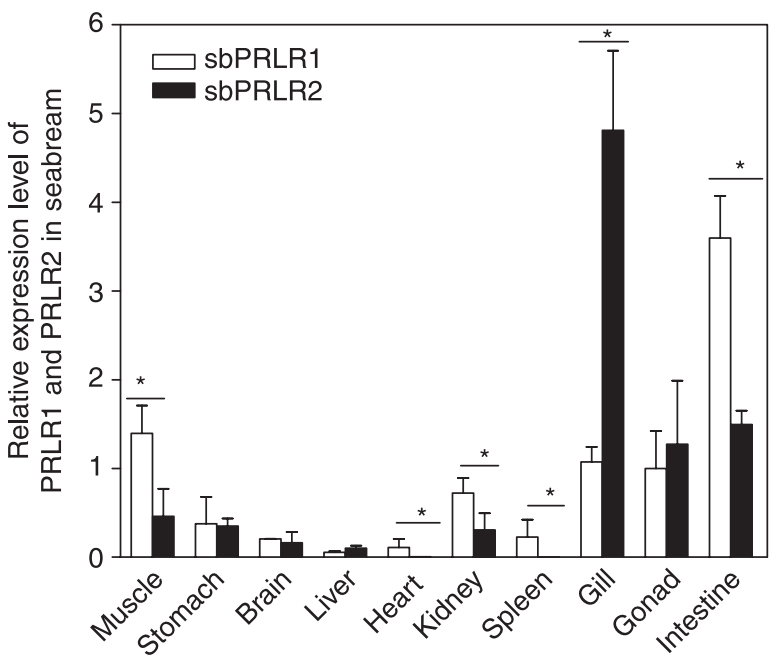

Figure 7 Distribution of PRLR1 and PRLR2 in seabream tissues. Results are normalized against $\beta$-actin expression and are expressed relative to the gonad PRLR1 level. Results are mean values \pm s.E.M. from six fishes. Significant difference, as revealed by unpaired $t$-test between sbPRLR1 and sbPRLR2 expression in each tissue type, is marked by asterisks $\left({ }^{*} P<0 \cdot 05\right)$.

\section{Discussion}

Genome analysis predicts the presence of two PRLR genes in fugu and zebrafish. This in silico prediction was experimentally substantiated by demonstrating the presence of two distinctly different PRLR cDNA sequences in both black seabream and

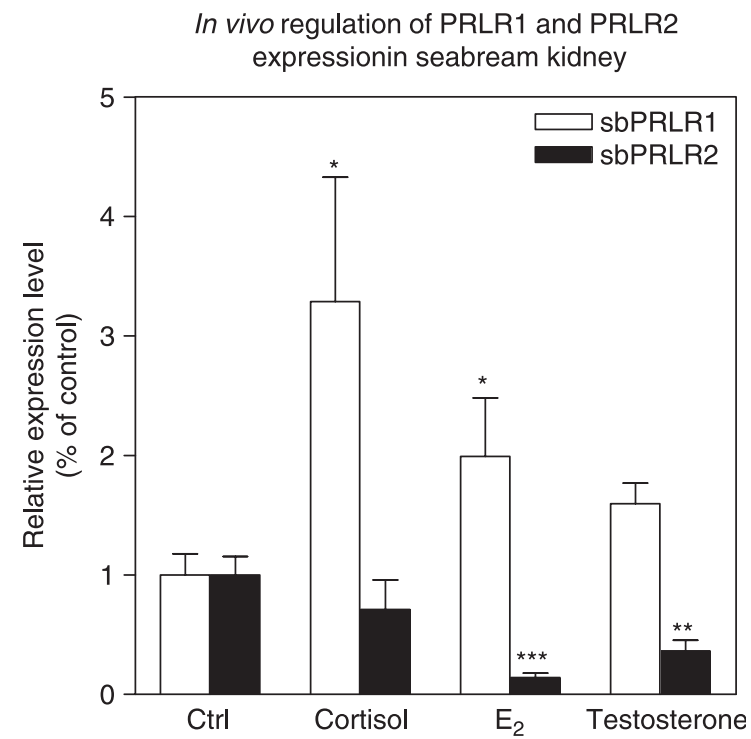

Figure 8 In vivo regulation of PRLR1 and PRLR2 expression in seabream kidney by hormone treatment. The hormone concentrations used were: cortisol $(5 \mu \mathrm{g} / \mathrm{g}), \mathrm{E}_{2}(2 \mu \mathrm{g} / \mathrm{g})$, and testosterone $(2 \mu \mathrm{g} / \mathrm{g})$. Results are mean values \pm s.E.M. from six independent fishes $\left({ }^{*} P<0 \cdot 05 ; * * P<0 \cdot 01 ; * * * P<0 \cdot 001\right.$ as compared with the respective control by one-way ANOVA).
Nile tilapia. Subsequent analysis of all the available PRLR sequences known to date revealed that there are indeed two clades of fish PRLR, which we call PRLR1 and PRLR2. In fact most of the PRLRs discovered so far in fish belong to the PRLR1 clade including those from goldfish (Tse et al. 2000), Nile tilapia (Sandra et al. 1995), halibut (Higashimoto et al. 2001), rainbow trout (Prunet et al. 2000), and carp (San Martin et al. 2006). The PRLR1 clade in fish is more akin in structure to the PRLRs found in other non-fish vertebrates. Nonetheless, there are also examples of previously discovered fish PRLRs which belong to the PRLR2 clade and these include those from gilthead seabream (Santos et al. 2001) and fugu (Lee et al. 2006). Without realizing that there are two types of PRLR in fish, the differences found in different fish PRLRs have been attributed to the diverse phylogeny of different fish species. Our data therefore represent the first report demonstrating the concomitant existence of two PRLR cDNA subtypes in a single fish species in different teleost lineages. This phenomenon is entirely different from the demonstration of two PRLRs found in carp (Cyprinus carpio; San Martin et al. 2006) in which both sequences belong to the PRLR1 clade, as shown in the phylogenetic analysis (Fig. 5). In view of the tetraploid nature of this fish, it is highly likely that other PRLR sequences belonging to the PRLR2 clade might also exist in carp, pending experimental proof. The same applies to other fish species in Fig. 5 in which only one subtype of the two PRLR clades has been discovered so far. Liu et al. (2006) did point out the presence of two genes for PRLR in zebrafish. However, they have neither reported the PRLR2 sequence nor demonstrated its functional significance. Moreover, they did not realize that it is a common phenomenon in teleosts.

Using black seabream as the animal model, we succeeded in obtaining the entire coding regions of the two PRLRs in this fish species. This renders parallel studies on these two receptors in a single fish species possible. Thus, these two receptors were functionally expressed in cultured eukaryotic cells in order to study their signaling events upon ligand stimulation. Real-time PCR methods were developed to enable simultaneous quantitative assessment of their expression levels. Moreover, the promoters of the respective genes have been cloned and studied in transfected cells by means of reporter gene assays. The two PRLR subtypes in seabream were shown to exhibit different patterns of tissue distribution, post-receptor signaling, and differential regulation by steroid hormones at the gene expression level as well as at the promoter level.

In the present study, the PRLR1 and PRLR2 sequences obtained feature the long form of mammalian PRLRs which contain an extracellular domain, a transmembrane domain, and an intracellular domain. Two pairs of conserved cysteine residues were found in fish PRLR1 and PRLR2, suggesting a similar hormone-binding site for the two receptor subtypes (Rozakis-Adcock \& Kelly 1992). In the extracellular domain, the conserved WS motif was found in both PRLR subtypes, indicating the possible significance of this motif in the two fish 
GAGAATGCTGCAGCTCTGTTTTGCTGTGAAGCTCCAGGAATGTTTTGAAACTTAACCTGACTTTCCCATAAGCATGGGGTTAAGTAGA STATX

TAATGACTGAATTTTTCTTTTTGGGTGAACGTATCCTTTAATGCATTAATCATATGATAATTGTATGATAGCTGATACAATCTCCTC -2285

CATTTGAGTGCTTTTATTTGTGATGATTTCAGCACATGTTGCTATATTTTAGCAATTCATGTCTTGTATGCGTGCTGATGCCCCTTTT -2197

TTTTTTGCCAGCCGTGCCCCTGAAACATCCGGAGTCCGTCACTGCGGTCTGTACAATTACCACTTTCCAGGAATTTCCAGTCCGTCCG -2109

NF-kappaB

CACGCATCTTTGAATGCATATGTGGGTATTTACGTCTGTGTCTGCAACATCTCTATAGGTGTGGCTTCCCAGAGAAATGAACACAA -2021

TGGGAGAAAAGGAAGTTCAGTTCAGGACACAATGATCGGACGCAGAAACAGATCCCTAAAGATCCCAGAAGGATTCTACAATTCATTT -1933

GGCTTTATGACTTTCCATAGTTTACAGCTTACTCATTGTGTTTACTCAAGGACAAAAGGTGCACTACCTTGTAGCCTTGTGCTATTTA -1845 CdxA

GATGGACGCACACTTAAGTACATGCAGTCTTCTCGGTGGGAACATCCTGGGGTTACAGCCTGCTGTAAATCAAATACTGCTCTACGTT ADR1

CTCTCCAGAGGATTTGTCTGACTGTTTATAATATTATGATTTTTTTTCTCCTTTTAGAGAATTAGAGCCTGCATAAAAGTGTGTATT XFD-2

AATCCTGTATGTATCAAACATTTTTAGTAGAGATAAACTCGCTCATATATACTAGTGTAAAAATAAGAGAGAGCAAGTCAGGGACACA -1581 NIT2

TTATGGAGGGCGGGGGGAGGGAGAGAGGGATGTCAATTACTGGGAGACCTCACTATGTTTACAGCTCCCAGTAAAGTAAAGAGGAACC -1493 ADR1 Dfd

AGTGAACACTTAGACACTATGTTAGGAGGTCAGGGCGTTCTGATCATCAAATCGTGTTTTACAATATTTGTTCATATGCAAAGCGTGT -1317

GRE $\frac{\text { ERE }}{\text { HNF-3b }}$

AAAAGTCACTAAGGTAGCAGCACAGGAATGACCATGTCATGTCCTGAGGAGAGGAGAATTGAATTTGTTGTATTATTAGATAGATAGA -1317 cap

TAGATAGATACTTTATTGATCCCGGAGGAAATCAAGTATTATATTATATTATATATTATTATATATTATAACATTCCTGATCTTATA -1229 CDP CR

ACCTTTAAGCTTCACATACACAGTTTTGAAATGTGGCTTTTATTGAGTGATGTAACTAAGAAATTACATCTGCCATCACTGATCATCA -1141 MATal HSF

GAATGAAAGTGCAAATCTAATTGATGATTAAATAACTTACTTTTAAAACATTTAAACATTTTTGTGGCTCTGATGCAAAGTAACTACT -1053

GACTAAAGGTATCAGTTGAACATAGTAAGTAAATATTACCCCTCCAAAATGTACTGAAAGTATAAAGTAACAGAAAATGGAAATACTT -965 XFD-1

TAGTGAACGTAGTTATTGTAGTTACATTCCATTACTTATACATACTTGCGTCTTTCTACTTCTACTCCATCACATCTCAGCCATTAGC

$-877$

CAGTTAATCAATAACTGTTTAAGTGATCTTTAATCTAAATACATTTCATAGCTCCAGCTTCACAAATGGAAATTGAGGCAAATTTGCT -789 c-Myb Nkx-2

TTTTCTTTTCAATTATCTTTTAGAAACATTCTTTTAATAATGGGCTAGGGTTAGGGTATTTTACAAACCAAAAAATTAATTGATTAAT

GCAGAAAATAATCAGGGGAATCAGTGGTAAGTTACTATTTTGTGACTATCATTTTTATAGTCATATTTCAAATGTAATGATATCTTTA -612 STRE

TTGTGTTATGAATATATATGTATGTATGTATATATTATAAAAAGGTATGTATAAAGGTAAACTCTTAGTTGGATACTAACACTCCCTC -524 CF2-II TATA

CACCACTGCACGGTTGCTTTAGGAGGAAATTGCACTGACCCTCATTCATTTCCTGGAAAGTTTCTAACCTCTAACCACTGCATGTGAC -436 STATx

ATCCAACCCCTGAAACACGCGCACACACACACACACACACACACACACAGAGGAATATATGATTAACCAATACAAAGCTCTACTGAAC -348

CACAGCTACCAGCCCTTGTTTATCTCTTTTCTTAGAGGTCAAACTCATCCAGCGTGACTGCTCATTGTATGGGCGTGGTTTAAACTGG -260 NIT2 ERE

ADR1

GGGTGGGAGGGGTCTATGCTCGTCCGCCACGCCCCCTCATAATGATAGCGCGGGCGGACTGAGAGGCGGTGGATGTTTCGACTTCTTG -172 Oct-1

TGGCAGACGCTCATGAGTGTACCATCGTCCCACCAGCCCGGTAAACCGGTTACACAAGTCGGTGAGTTCAGTTCGTTCTTCTGGGAGC -84 CGCCAGTGGACCCAGCACGGCCAGAACACCGAGGGAGAGACTGAAAGCTGCTGTCAGCTGAAGCTGAGGCTTTGTGGTGCCACGATG

Figure 9 Sequence analysis of the $5^{\prime}$-flanking region of the $S B P R L R 1$ gene. The putative binding sites for transcription factors are underlined. These transcription factor-binding sites were identified using both TFSEARCH (http://www.cbrc.jp/research/db/ TFSEARCH.html) and TESS (http://www.cbil.upenn.edu/cgi-bin/tess/tess?RQ=SEA-FR-QueryS). 
AAATAAATGAAGAAATAATAATATAACAATAACAAACATTACTTTTATTATTGTTATTATAGAGATTTTGAATCTTAAGGGTCTCTCTGGA -2179 HSF Sox-5

TTGTGTAACAAGTAGCTGGAAATGTGATTAATACTGTTCTTATAGTCAGTATTGTGTTTTGTAGATTGAGTGTCCTCCTCAAAAAATGTTTA

TTCTGATGAGAAAGGACACCTGAAATGTGAGGCTGCCTAAGATGTTCCCCTAAAACTGAAAAGCCAACACCTTGAGAATTAGAGGATAAAA -1993

STATx

TCACCCAAAAATGGAAATTCATCATCTACTTTTGAAGTTCACAAAAATGTCTACAGCTTCACAGCAAAACAGTCTTGCAGCATTTTCCTAAAC -1900

SRY

AACTTGTAGATGGGGACCTACATTTACAAATGAGGGGTCCTGTCATTGTCCCATGAGAACCATCTCAAACACACTCCATCTCCACCGAGTGA -1807

GAGAGAGAGGTCAGTGCAATGCTCAGCTGATTTACTTTTGTACAGTGTAGCATTCATATATATACTTTCTTTGCATTTTGCTTTTTTTCAACA

ERE AP4

CF2-II

GGTCAGGTTAGAATACAGACATGCACTGTCATGAAACCAGCCAGTGTATGTAGTGGGATGCGGGTGTGGATCGTAGAAGATTAACAAGGTTAA ERE

$\frac{\text { CAATGATGTGCACACATATTAAACCCCAACGATAAAACAATCATGTTGACTGTAAGAATATGCCCTCCTCACTGCACCCTCAGGCCAGACAGG }}{\text { Sox-5 }}$

СTAACTCCATCCСTGGTAGAAGGGTGGAGTGGAAAACTACCAGCССTGTTGTTCACACGCTAGTGCTCAGTCAGCTGTGTACAAATCTTTGCT

GCTGTGCAAGTCAACCTGTGTGATCCTCAACCCTGGCTTTAAAGAAAAATCACAGTGGTGGTTATCATTGACAGCTGATGTTCAGTCATTTTT

AATTTGACСATTTTCATATCAAAAAGGTAAAAAAGGTTTGTGTTTTGGTGCACACTTTTTGTTCTTATCAAGGTGGAAAAAATGCTGCAGCTG

GATA-X

САTTTATTTCCСАСTTATTATTTGTAACATTTGCCACAACTACTCTGTAAAAAAGTATAGCTTACAGTCGCTAGTGTGGCATCAGTCTTGC

cap

TTAGTCTTGTTGGCTCTGCATTGCATTTGTACTGTAGAATATCAATAGATACTACAGATGAAGAACGTTGCACCAGAGATGATTTGATTTC HSF

CGTGAATTCCATAAATCTGTTCATCATTTATTTGTGTTGGAGTTTTTTGAGGTCAACTTTTTTCTTGCCAATTGCTCATTTGACATCAGTTGA ERE

ATAAAGAAGCAAAAATGTAAAAAAAAAAAAAAAAAATGTTGCAGAAAAAAAAGTTTGTGGAGATGAAAAGGGGGAAAAATAGGGTGGCACTTT

GCTCAGGAGGGAGAGCGGGTTGTACAGAATTCGGAAGGTCGCTGGTTTGACTCCCAGGATCCTCTGGCTGCAGGTCGAAGCATCCATGAGCAA

GAAACTGAGCCCTGAGTTGCTCCTGATGAGCAGGTGGGCAGCTGGCATGGCAACAGCCAACTCCATCAGTGTGTGAATGGCTGAATTTCCTA

AGTCAGTACATAGgGAAAAGAGTAAAAGCAATGGAAAGAAAGAAAATAGAAATAGAAAAATAACACAAAAATGAATAAATTCAGAAATGTATG

ATTTATTTAAAAAAAAAAGAATAGATGAATTAAAAAATGAAAATTATTAATTGGACTGTAAATTAATTTGACAATTAAAAAGGTAATAAACA GR

CGCAAACAATAATTAATGAATACATTTTATTTATTTTTTAAATCATTGGTGCATTTAACAGCACGCCAGTTTATCTATTAAGTAGTGTATTT -41 Sox-5

NIT2

ACTTATTCATTACTACCAATATTAGTATTATTTTGATTCAGACAGTTTTGGTTCTCCGTACTAGCTCGCTTGGTAAAGTATAATCTGCCGGTG

GGGCAGAAATTCGGTTTATAATAACCCAGAATAAAGCAGGAGTGACACATCTCGGTTACGCCACGCTCCCTCGGGTAGAGGAAATTTGACGG -226

AATCAAGCСССТССССTCGTGGCTCAGCTCACСTCTCTTCCTCATTACATACTCCACCTCAGGTGACAGATGCGAGGGTTACCAGGTGTGA -133

GAAGGTCGTCGACGACAATGTTGCGCCGCAGGAGGATTCTGTTCGCTCTGTCGACAGCAGCAACATGGAGTTGAGTGACTCAGTGGTGTAGG

$-40$

TCGACACTCAGCTGGACACACTGCGGGACAGTTGGTGCCATG

Figure 10 Sequence analysis of the $5^{\prime}$-flanking region of the $s b P R L R 2$ gene. The putative binding sites for transcription factors are underlined. These transcription factor-binding sites were identified using TFSEARCH (http://www.cbrc.jp/research/db/TFSEARCH.html) and TESS (http://www.cbil.upenn.edu/cgi-bin/tess/tess?RQ=SEA-FR-QueryS).

receptors. In a previous study performed on mammalian PRLR (Rozakis-Adcock \& Kelly 1992), this region was suggested to provide a target site for interaction with some accessory proteins necessary for the formation of a highaffinity receptor complex. Whether the same holds true for the fish receptors remains to be established. The transmembrane domain of PRLR1 and PRLR2 comprises about 24 a, which are similar to other PRLRs. Box 1 is the most conserved motif in the intracellular domain, which is consistent with its importance in the signal transduction 
A

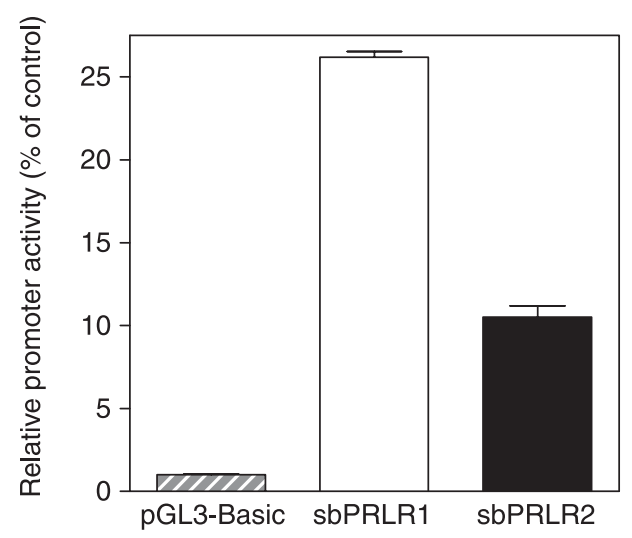

$\mathrm{C}$

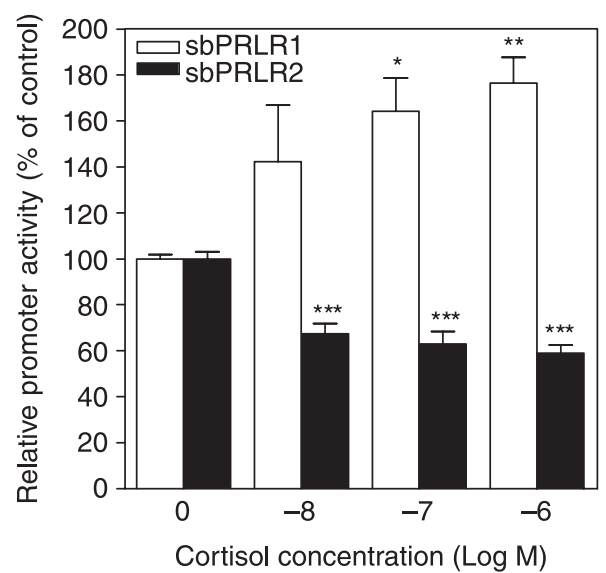

B

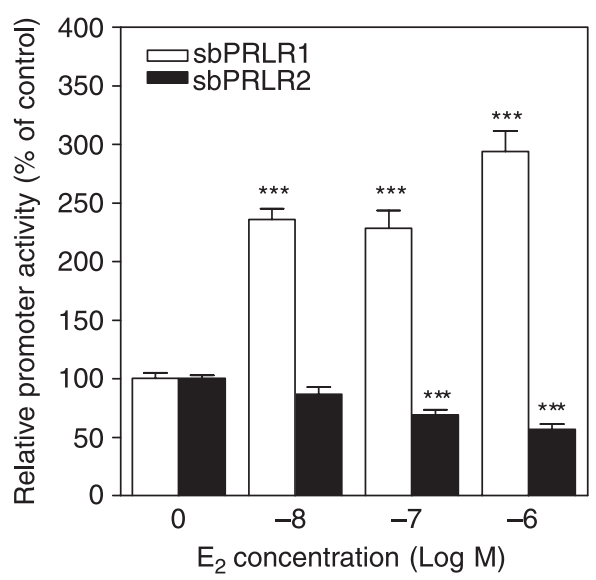

D

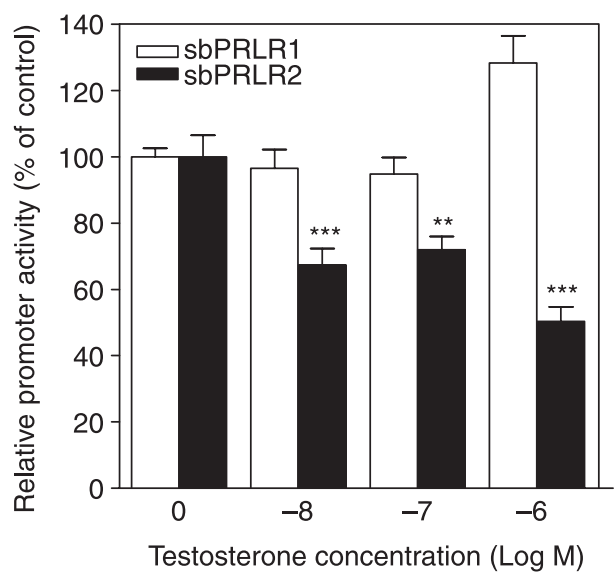

Figure 11 (A) The basal activities of $s b P R L R 1$ and $s b P R L R 2$ promoters in transfected GAKS cells. The cells were transfected with $0.5 \mu \mathrm{g}$ pGL3-basic vector containing either the $s b P R L R 1$ or sbPRLR2 gene promoter construct and $0.05 \mu \mathrm{g}$ pRL-CMV. The empty pGL3-Basic vector was used as a control. The transfected cells were incubate with the medium without any hormone for $24 \mathrm{~h}$. Relative promoter activities are expressed as a percentage of the control. (B-D) The effect of $\mathrm{E}_{2} /$ cortisol/testosterone on the $s b P R L R 1$ and $s b P R L R 2$ gene promoter activities in transfected GAKS cells respectively. The cells were transfected with $0.5 \mu \mathrm{g}$ pGL3-basic vector containing either the $s b P R L R 1$ or $s b P R L R 2$ gene promoter construct and $0.05 \mu \mathrm{g}$ pRL-CMV. The

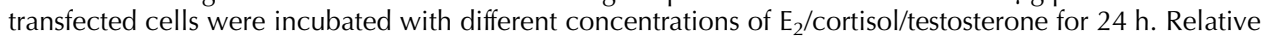
promoter activities (i.e. those under hormonal stimulation) are expressed as a percentage of the control in the absence of the hormones (i.e. the basal promoter activity without hormonal stimulation). Results are mean values \pm S.E.M. $\left(n=9 ;{ }^{*} P<0 \cdot 5,{ }^{* *} P<0 \cdot 01,{ }^{* * *} P<0 \cdot 001\right.$ as compared with the respective control by one-way ANOVA).

pathway (Dinerstein et al. 1995, Pezet et al. 1997). A typical Box 2 is absent in PRLR2 (Fig. 4). To date, the function of Box 2 in PRLR is not well defined. Thus, the significance of the absence of this motif awaits further elucidation. In addition, PRLR 2 has four other deletions in the intracellular domain as compared with PRLR1, contributing to the loss of some intracellular tyrosine residues (Fig. 4). For sbPRLR1, there are 11 intracellular tyrosine residues but for sbPRLR2, there are only 7 such tyrosine residues. Similarly, tilapia PRLR1 has 11 intracellular tyrosine residues, whereas tilapia
PRLR2 has only 7 tyrosine residues in the intracellular domain (Fig. 3). Due to the importance of these intracellular tyrosine residues in the signal transduction of cytokine receptors, such deletions might also result in changes in the biological activity of the receptor.

Expression of the cloned receptors in cultured eukaryotic cells followed by functional assays using specific promoterdriven reporter gene assays in the present study indicated that sbPRLR1 and sbPRLR2 are indeed functional entities capable of transducing post-receptor signaling events. It has 
been reported previously that association of Janus kinase (JAK) 2 to the Box 1 region is essential in mediating the signal transduction of mammalian PRLRs, followed by the activation of $\mathrm{c}$-fos and $\beta$-casein expression (Lesueur et al. 1991, Berlanga et al. 1995). In our study, both sbPRLR1 and sbPRLR 2 could activate the $c$-fos and $\beta$-casein promoters upon receptor stimulation. The two receptors exhibited similar activity towards activating the $\mathrm{c}$-fos promoter but sbPRLR1 appeared to be more potent than sbPRLR2 in activating the $\beta$-casein promoter. In addition, only sbPRLR1 but not sbPRLR 2 could activate the Spi 2.1 promoter. Such differences probably reflect the innate abilities of the receptors in mediating the signal transduction processes as they exhibited a very similar expression level in the transfected cells (Fig. 6D). The ligand specificity results indicated that the receptors could respond to PRL, but not to GH and SL. These results are consistent with the fact that the two receptors have a similar extracellular ligand-binding domain but with much differences in the intracellular domain. In fact, the Spi 2.1 pathway that we have demonstrated for sbPRLR1 is a novel one for PRL signaling. Spi 2.1 promoter activation has been demonstrated for mammalian GH receptor (GHR; Dinerstein et al. 1995, Gong et al. 1998) as well as for fish GHR (Tse et al. 2003, Jiao et al. 2006). To our knowledge, there is no previous report on the Spi pathway for PRLR.

$\mathrm{PRL} / \mathrm{SL} / \mathrm{GH}$ belong to the same cytokine family. They share many common features and so do their receptors. Recently, two SL receptor (SLR) sequences have been cloned from trout and medaka fish, and they are similar to the GHR in teleosts (Fukada et al. 2005, Fukamachi et al. 2005). This raises the question of whether the PRLRs cloned in the present study may also cross-react with GH or SL. The results in Fig. 6 clearly demonstrated that the cloned receptors could respond to the ligands in a specific manner indicating that they are truly PRLRs. This is in line with the phylogenetic analysis results showing that the cloned sequences are PRLRs but not GHRs or SLRs.

It should be mentioned that we have adopted a heterologous system in our study, viz. using salmon hormones to analyze the functional activity of seabream PRLRs in a human cell line. Since HEK293 cell line is a wellcharacterized cell line used in the functional expression of similar receptors, we have employed this established system to express our cloned receptors in order to avoid other uncertainties. The use of heterologous hormones in our system is not the most ideal. The availability of wellcharacterized and highly purified pituitary hormones from salmon and the lack of availability of the same hormones from seabream render this a feasible alternative. The message from the ligand specificity studies is clear although it could be argued that the amplitude of the response could be higher if one uses homologous hormones.

Real-time PCR was developed for the accurate quantitation of both receptors in seabream tissues. The sbPRLR1 and sbPRLR2 were found to be expressed in tissues implicated in the control of water and ion balance including the gill, intestine, and kidney (Fig. 7), in line with the fact that these are the target organs of PRL. Interestingly, the expression of sPRLR1 is higher than that of sbPRLR2 in most tissues with the notable exception of the gill in which the expression of sPRLR2 is several fold higher than that of sbPRLR1, suggestive of a particular biological role of sbPRLR2 in the gill of the fish. The seabream gonad contains similar levels of both receptors, in line with the important role of PRL in reproduction (Bole-Feysot et al. 1998). The wide distribution of the receptors in different tissues of seabream is consistent with the multifaceted roles of PRL in fish. It is interesting to note that the black seabream muscle was found to express measurable amounts of PRLR, but not in the liver. This is in general agreement with previous finding in gilthead seabream (Santos et al. 2001), the significance of which is unknown at the moment. This diverge tissue distribution patterns of the two receptors in seabream indicate that these receptor paralogs in fish probably possess some different biological functions.

Reports on the actions of steroid hormones on the gene expression of PRLR in fish are scanty. In this study, the effects of steroid hormones on sbPRLR 1 and sbPRLR 2 expression were studied both in fish administered with steroid hormones in vivo and at the gene promoter level in vitro. The functionalities of the putative promoter regions of the sbPRLR1 and sbPRLR2 genes were tested by transient transfection in cultured cells by a reporter gene assay. The $5^{\prime}$-flanking region of the $s b P R L R 1$ gene exhibited about 30 -fold induction of the promoter activity, while the $5^{\prime}$ flanking region of the $s b P R L R 2$ gene showed about 10 -fold increase in the promoter activity as compared with the promoterless construct.

In our study, the expression level of sbPRLR1 in the kidney was up-regulated, while the sbPRLR2 was downregulated by $E_{2}$ treatment of the fish (Fig. 8). Results of the promoter studies correlated with the gene expression studies as treatment of the transfected GAKS cells with increasing dose of $\mathrm{E}_{2}$ activated the $s b P R L R 1$ gene promoter activity but inhibited the $s b P R L R 2$ gene promoter activity (Fig. 11B). Estrogen exerts its transcriptional regulation by binding to the ER and targeting to the estrogen-responsive element (ERE), which consists of a palindromic repeat of the $5^{\prime}$-TGACCT- $3^{\prime}$ half site (Zilliacus et al. 1995). By searching the sequences in our $s b P R L R$ gene promoters, we have also located several such sequences (Figs 9 and 10). On the other hand, a new transcription mechanism that does not involve the ERE was recently found (Dong et al. 2006). Since $E_{2}$ could differentially alter the gene expression levels of sbPRLR1 and sbPRLR2 as well as the promoter activities, it is interesting to know whether the transcriptional regulation for these two receptors involves different mechanisms. Some other studies indicated that the PRLR expression level could be differently regulated by $\mathrm{E}_{2}$ at different developmental stages in different species. In the gonad of adult gilthead seabream, the transcript level of PRLR, which is a PRLR2 according to the phylogenetic analysis (Fig. 5), increased significantly 
(50-fold) after $\mathrm{E}_{2}$ treatment, but a 24-fold decrease was found in the juvenile gonad (Cavaco et al. 2003). However, in mammals, $E_{2}$ stimulates the expression of PRLR in various tissues (Cheng et al. 1984, Tsim et al. 1985, Barash et al. 1992, Cassy et al. 2000). The mechanism for these differences awaits further studies to unravel.

The hormonal control of adaptation to seawater involves the pituitary interrenal axis with cortisol as the final steroid product (McCormick 2001). In this study, it was demonstrated that treatment of fish in vivo by cortisol increased the expression of sbPRLR1 but had no effect on the expression of sbPRLR2 (Fig. 8). At the promoter level, the sbPRLR1 gene promoter activity was increased, in contrast to the decreased $s b P R L R 2$ gene promoter activity when challenged with increasing dose of cortisol (Fig. 11C). Glucocorticoids can regulate gene transcription by multiple mechanisms including direct GR binding to a gene promoter and those that involve GR acting on a gene promoter via interaction with a DNA-bound transcription factor (Newton 2000). Positive and negative glucocorticoid-responsive elements (GRE) have been identified in several gene promoters such as the $\mathrm{Na}^{+} / \mathrm{K}^{+}$-ATPase $\beta 1$ subunit (Derfoul et al. 1998), Dax-1 (Gummow et al. 2006), and glucose-6-phosphatase catalytic subunit (Vander Kooi et al. 2005). However, negative GRE in fish have never been reported in any gene promoter. In our case, only positive GRE were identified (Figs 9 and 10) because negative GRE do not often match the consensus sequence (Vander Kooi et al. 2005). Under the stimulation of cortisol, the $s b P R L R 1$ and $s b P R L R 2$ gene promoters exhibited very different luciferase activity patterns. The mechanisms adopted by these two promoters in their cortisol response remain to be elucidated.

Apart from estrogen (Guzman et al. 2004), testosterone was also demonstrated to regulate osmoregulation through $\mathrm{Na}^{+}$/ $\mathrm{K}^{+}$-ATPase. In a recent publication by Sangiao-Alvarellos et al. (2006), the role of testosterone on osmoregulation was studied on the euryhaline teleost gilthead seabream. Fish implanted with testosterone ( $5 \mu \mathrm{g} / \mathrm{g}$ body weight), the kidney $\mathrm{Na}^{+} / \mathrm{K}^{+}$-ATPase activity was enhanced on the first day but diminished by day 3. In our study, testosterone was demonstrated to regulate the expression of PRLRs in fish. The expression of sbPRLR1 was not significantly changed but the expression of sbPRLR2 was down-regulated (Fig. 8). These results were consistent with our promoter studies in which the $s b P R L R 1$ gene promoter activity remained unchanged but the $s b P R L R 2$ gene promoter activity was inhibited (Fig. 11D). The AR-mediated gene expression suppression might be due to the presence of a co-repressor of the ligand-bound AR, such as cyclin D1 (Burd et al. 2005) or Daxx (Lin et al. 2004). The discovery of these regulators could provide us with a more comprehensive explanation of the transcriptional regulation of PRLRs. In our study, only sbPRLR2 could respond to the exogenous androgen treatment suggesting that distinct physiological functions exist for these two receptors.

Evidence suggests that fish underwent a fish-specific genome duplication which accounts for the presence of more duplicated genes in teleosts than in tetrapods (Hoegg et al. 2004, Meyer \& Van de Peer 2005). The hypothesis is becoming more widely accepted based on evidence from the recent genome annotation work of the fugu and zebrafish genome sequences. In the present study, two PRLR subtypes encoded by two different genes were found in four different teleost species, viz. black seabream, Nile tilapia, fugu, and zebrafish. The results provide additional evidence for a genome duplication in the Actinopterygia (ray-finned fishes). Duplicated genes in a genome can only be maintained if they acquire a new function or maintain only some functions of the ancestral gene (Force et al. 1999). In this study, we have demonstrated that the two receptors exhibited distinct differences in several aspects. These functional differences probably explain and justify the preservation and coexistence of both PRLR subtypes during teleostean evolution after the genome duplication.

\section{Acknowledgements}

We thank Dr Nils Billestrup of the Hagedorn Research Institute in Denmark for providing the rat Spi 2.1 promoter construct, and Dr K L Yu at the University of Hong Kong for providing the rat $\beta$-casein and human $c$-fos promoter constructs. We are also grateful to Dr Penny Swanson at the Northwest Fisheries Science Centre of USA for providing us with the purified sGH, sPRL and sSL preparations. The following granting agencies are gratefully acknowledged: the Research Grants Council of the Hong Kong Government (Project No. CUHK4418/06M), The Chinese University of Hong Kong Direct Grants (Project No. 2041259 and 2041181), the Guangdong Provincial Scientific and Technical Program (No. 2004A20105001), the Leading Programme of State Education Ministry (No. 2004-104161), the Chongqing Natural Science Foundation (No. CSTC2004BB8450), and the Free Exploration Fund of the Key Laboratory of Ecoenvironments in the Three Gorges Reservoir Region (Ministry of Education) (No. 124470-20500312). The sequences reported in this paper have been deposited in the GenBank database at NCBI and are assigned the following accession numbers: EF429092, EF429093 and EF429094. The authors declare that there is no conflict of interest that would prejudice the impartiality of this scientific work.

\section{References}

Barash I, Madar Z \& Gertler A 1992 Short-term in vivo regulation of prolactin receptors in the liver, testes, kidneys, and mammary gland of rats. Receptor 2 39-44.

Berlanga JJ, Fresno Vara JA, Martin-Perez J \& Garcia-Ruiz JP 1995 Prolactin receptor is associated with c-src kinase in rat liver. Molecular Endocrinology 9 1461-1467.

Bole-Feysot C, Goffin V, Edery M, Binart N \& Kelly PA 1998 Prolactin (PRL) and its receptor: actions, signal transduction pathways and phenotypes observed in PRL receptor knockout mice. Endocrine Reviews 19 225-268. 
Burd CJ, Petre CE, Moghadam H, Wilson EM \& Knudsen KE 2005 Cyclin D1 binding to the androgen receptor (AR) NH2-terminal domain inhibits activation function 2 association and reveals dual roles for AR corepression. Molecular Endocrinology 19 607-620.

Cassy S, Charlier M, Belair L, Guillomot M, Laud K \& Djiane J 2000 Increase in prolactin receptor (PRL-R) mRNA level in the mammary gland after hormonal induction of lactation in virgin ewes. Domestic Animal Endocrinology 18 41-55.

Cavaco JEB, Santos CRA, Ingleton PM, Canario AVM \& Power DM 2003 Quantification of prolactin (PRL) and PRL receptor messenger RNA in gilthead seabream (Sparus aurata) after treatment with estradiol-17 $\beta$. Biology of Reproduction 68 588-594.

Celius T, Matthews JB, Giesy JP \& Zacharewski TR 2000 Quantification of rainbow trout (Oncorhynchus mykiss) zona radiata and vitellogenin mRNA levels using real-time PCR after in vivo treatment with estradiol-17 $\beta$ or $\alpha$-zearalenol. Journal of Steroid Biochemistry and Molecular Biology 75 109-119.

Chan CB \& Cheng CHK 2004 Identification and functional characterization of two alternatively spliced growth hormone secretagogue receptor transcripts from the pituitary of black seabream Acanthopagrus schlegeli. Molecular and Cellular Endocrinology 214 81-95.

Chan CB, Leung PK, Wise H \& Cheng CHK 2004 Signal transduction mechanism of the seabream growth hormone secretagogue receptor. FEBS Letters 577 147-153.

Cheng CHK, Tsim KWK, Wai MK \& Pak RCK 1984 Rat hepatic prolactin receptor: pubertal exposure to sex steroids alters responsivity to testosterone. International Journal of Peptide and Protein Research 23 521-527.

Derfoul A, Robertson NM, Lingrel JB, Hall DJ \& Litwack G 1998 Regulation of the human $\mathrm{Na} / \mathrm{K}$-ATPase $\beta 1$ gene promoter by mineralocorticoid and glucocorticoid receptors. Journal of Biological Chemistry 273 20702-20711.

Dinerstein H, Lago F, Goujon L, Ferrag F, Esposito N, Finidori J, Kelly PA \& Postel-Vinay MC 1995 The proline-rich region of the GH receptor is essential for JAK2 phosphorylation, activation of cell proliferation, and gene transcription. Molecular Endocrinology 9 1701-1707.

Dong J, Tsai-Morris CH \& Dufau ML 2006 A novel estradiol/estrogen receptor $\alpha$-dependent transcriptional mechanism controls expression of the human prolactin receptor. Journal of Biological Chemistry 281 18825-18836.

Force A, Lynch M, Pickett FB, Amores A, Yan YL \& Postlethwait J 1999 Preservation of duplicate genes by complementary, degenerative mutations. Genetics 151 1531-1545.

Freeman ME, Kanyicska B, Lerant A \& Nagy G 2000 Prolactin: structure, function, and regulation of secretion. Physiological Reviews 80 1523-1631.

Fukada H, Ozaki Y, Pierce AL, Adachi S, Yamauchi K, Hara A, Swanson P \& Dickhoff WW 2005 Identification of the salmon somatolactin receptor, a new member of the cytokine receptor family. Endocrinology 146 2354-2361.

Fukamachi S, Yada T \& Mitani H 2005 Medaka receptors for somatolactin and growth hormone: phylogenetic paradox among fish growth hormone receptors. Genetics 171 1875-1883.

Gong TW, Meyer DJ, Liao J, Hodge CL, Campbell GS, Wang X, Billestrup N, Carter-Su C \& Schwartz J 1998 Regulation of glucose transport and c-fos and egr-1 expression in cells with mutated or endogenous growth hormone receptors. Endocrinology 139 1863-1871.

Gummow BM, Scheys JO, Cancelli VR \& Hammer GD 2006 Reciprocal regulation of a glucocorticoid receptor-steroidogenic factor-1 transcription complex on the Dax-1 promoter by glucocorticoids and adrenocorticotropic hormone in the adrenal cortex. Molecular Endocrinology 20 2711-2723.

Guzman JM, Sangiao-Alvarellos S, Laiz-Carrion R, Miguez JM, Martin del Rio Mdel P, Soengas JL \& Mancera JM 2004 Osmoregulatory action of $17 \beta$-estradiol in the gilthead seabream Sparus auratus. Journal of Experimental Zoology. Part A, Comparative Experimental Biology 301 828-836.

Harris J \& Bird DJ 2000 Modulation of the fish immune system by hormones. Veterinary Immunology and Immunopathology 77 163-176.

Higashimoto Y, Nakao N, Ohkubo T, Tanaka M \& Nakashima K 2001 Structure and tissue distribution of prolactin receptor mRNA in Japanese flounder (Paralichtys olivaceus): conserved and preferential expression in osmoregulatory organs. General and Comparative Endocrinology 123 170-179.
Hoegg S, Brinkmann H, Taylor JS \& Meyer A 2004 Phylogenetic timing of the fish-specific genome duplication correlates with the diversification of teleost fish. Journal of Molecular Evolution 59 190-203.

Jiao B, Huang X, Chan CB, Zhang L, Wang D \& Cheng CHK 2006 The co-existence of two growth hormone receptors in teleost fish and their differential signal transduction, tissue distribution and hormonal regulation of expression in seabream. Journal of Molecular Endocrinology 36 23-40.

Kitta K, Makino M, Oshima N \& Bern HA 1993 Effects of prolactins on the chromatophores of the tilapia, Oreochromis niloticus. General and Comparative Endocrinology 92 355-365.

Lee KM, Kaneko T \& Aida K 2006 Prolactin and prolactin receptor expressions in a marine teleost, pufferfish Takifugu rubripes. General and Comparative Endocrinology 146 318-328.

Lesueur L, Edery M, Ali S, Paly J, Kelly PA \& Djiane J 1991 Comparison of long and short forms of the prolactin receptor on prolactin-induced milk protein gene transcription. PNAS $\mathbf{8 8} 824-828$.

Lin DY, Fang HI, Ma AH, Huang YS, Pu YS, Jenster G, Kung HJ \& Shih HM 2004 Negative modulation of androgen receptor transcriptional activity by Daxx. Molecular and Cellular Biology 24 10529-10541.

Liu NA, Liu Q, Wawrowsky K, Yang Z, Lin S \& Melmed S 2006 Prolactin receptor signaling mediates the osmotic response of embryonic zebrafish lactotrophs. Molecular Endocrinology 20 871-880.

Livak KJ \& Schmittgen TD 2001 Analysis of relative gene expression data using real-time quantitative PCR and the $2^{-\Delta \Delta \mathrm{Ct}}$ method. Methods $25402-408$.

Manzon LA 2002 The role of prolactin in fish osmoregulation: a review. General and Comparative Endocrinology 125 291-310.

McCormick SD 2001 Endocrine control of osmoregulation in teleost fish. American Zoologist 41 781-794.

Meyer A \& Van de Peer Y 2005 From 2R to 3R: evidence for a fish-specific genome duplication (FSGD). Bioessays 27 937-945.

Newton R 2000 Molecular mechanisms of glucocorticoid action: what is important? Thorax 55 603-613.

Pezet A, Buteau H, Kelly PA \& Edery M 1997 The last proline of Box 1 is essential for association with JAK2 and functional activation of the prolactin receptor. Molecular and Cellular Endocrinology 129 199-208.

Prunet P, Sandra O, Le Rouzic P, Marchand O \& Laudet V 2000 Molecular characterization of the prolactin receptor in two fish species, tilapia Oreochromis niloticus and rainbow trout Oncorhynchus mykiss: a comparative approach. Canadian Journal of Physiology and Pharmacology 78 1086-1096.

Le Rouzic P, Sandra O, Grosclaude J, Rentier-Delrue F, Jolois O, Tujague M, Pakdel F, Sandowski Y, Cohen Y, Gertler A et al. 2001 Evidence of rainbow trout prolactin interaction with its receptor through unstable homodimerisation. Molecular and Cellular Endocrinology 172 105-113.

Rozakis-Adcock M \& Kelly PA 1992 Identification of ligand binding determinants of the prolactin receptor. Journal of Biological Chemistry 267 7428-7433.

de Ruiter AJ, Wendelaar Bonga SE, Slijkhuis H \& Baggerman B 1986 The effect of prolactin on fanning behavior in the male three-spined stickleback, Gasterosteus aculeatus L. General and Comparative Endocrinology 64 273-283.

Sambrook J, Fritsch EF \& Maniatis T 1989 Molecular Cloning - A Laboratory Manual. Edn 2, Cold Spring Harbor, NY: Cold Spring Harbor Laboratory Press.

Sandra O \& Prunet P 1998 A single prolactin receptor form in tilapia species? Annals of the New York Academy of Sciences 839 172-176.

Sandra O, Sohm F, de Luze A, Prunet P, Edery M \& Kelly PA 1995 Expression cloning of a cDNA encoding a fish prolactin receptor. PNAS 92 6037-6041.

Sangiao-Alvarellos S, Polakof S, Arjona FJ, Garcia-Lopez A, Martin del Rio MP, Martinez-Rodriguez G, Miguez JM, Mancera JM \& Soengas JL 2006 Influence of testosterone administration on osmoregulation and energy metabolism of gilthead seabream Sparus auratus. General and Comparative Endocrinology 149 30-41.

San Martin R, Hurtado W, Quezada C, Reyes AE, Vera MI \& Krauskopf M 2006 Gene structure and seasonal expression of carp fish prolactin short receptor isoforms. Journal of Cellular Biochemistry 100 970-980.

Santos CRA, Ingleton PM, Cavaco JEB, Kelly PA, Edery M \& Power DM 2001 Cloning, characterization, and tissue distribution of prolactin receptor in the seabream (Sparus aurata). General and Comparative Endocrinology 121 $32-47$. 
Thompson JD, Gibson TJ, Plewniak F, Jeanmougin F \& Higgins DG 1997 The CLUSTAL_X windows interface: flexible strategies for multiple sequence alignment aided by quality analysis tools. Nucleic Acids Research 25 4876-4882.

Tse DLY, Chow BKC, Chan CB, Lee LTO \& Cheng CHK 2000 Molecular cloning and expression studies of a prolactin receptor in goldfish (Carassius auratus). Life Sciences 66 593-605.

Tse DLY, Tse MCL, Chan CB, Deng L, Zhang WM, Lin HR \& Cheng CHK 2003 Seabream growth hormone receptor: molecular cloning and functional studies of the full-length cDNA, and tissue expression of two alternatively spliced forms. Biochimica et Biophysica Acta 1625 64-76.

Tsim KWK, Pak RCK \& Cheng CHK 1985 Prolactin receptor in rat liver: sex difference in estrogenic stimulation and imprinting of the responsiveness to estrogen by neonatal androgen in male rats. Molecular and Cellular Endocrinology 40 99-105.

Vander Kooi BT, Onuma H, Oeser JK, Svitek CA, Allen SR, Vander Kooi CW, Chazin WJ \& O'Brien RM 2005 The glucose-6-phosphatase catalytic subunit gene promoter contains both positive and negative glucocorticoid response elements. Molecular Endocrinology 19 3001-3022.

Vong QP, Chan KM \& Cheng CHK 2003 Quantification of common carp (Cyprinus carpio) IGF-I and IGF-II mRNA by real-time PCR: differential regulation of expression by GH. Journal of Endocrinology 178 513-521.
Yada T, Muto K, Azuma T, Hyodo S \& Schreck CB 2005 Cortisol stimulates growth hormone gene expression in rainbow trout leucocytes in vitro. General and Comparative Endocrinology 142 248-255.

Yeung CM, Chan CB \& Cheng CHK 2004 Isolation and characterization of the $5^{\prime}$-flanking region of the growth hormone secretagogue receptor gene from black seabream Acanthopagrus schlegeli. Molecular and Cellular Endocrinology 223 5-15.

Yeung CM, Chan CB, Woo NYS \& Cheng CHK 2006 Seabream ghrelin: cDNA cloning, genomic organization and promoter studies. Journal of Endocrinology 189 365-379.

Zilliacus J, Wright AP, Carlstedt-Duke J \& Gustafsson JA 1995 Structural determinants of DNA-binding specificity by steroid receptors. Molecular Endocrinology 9 389-400.

Received in final form 8 April 2007

Accepted 11 May 2007

Made available online as an Accepted Preprint

21 May 2007 